\title{
Single-valued neutrosophic Einstein interactive aggregation operators with applications for material selection in engineering design: case study of cryogenic storage tank
}

\author{
Hafiz Muhammad Athar Farid ${ }^{1}$ (D) $\cdot$ Muhammad Riaz $^{1}$
}

Received: 12 August 2021 / Accepted: 17 December 2021 / Published online: 15 January 2022

(c) The Author(s) 2022

\begin{abstract}
Single-valued neutrosophic sets (SVNSs) and their application to material selection in engineering design. Liquid hydrogen is a feasible ingredient for energy storage in a lightweight application due to its high gravimetric power density. Material selection is an essential component in engineering since it meets all of the functional criteria of the object. Materials selection is a time-consuming as well as a critical phase in the design process. Inadequate material(s) selection can have a detrimental impact on a manufacturer's production, profitability, and credibility. Multi-criteria decision-making (MCDM) is an important tool in the engineering design process that deals with complexities in material selection. However, the existing MCDM techniques often produce conflicting results. To address such problems, an innovative aggregation technique is proposed for material selection in engineering design based on truthness, indeterminacy, and falsity indexes of SVNSs. Taking advantage of SVNSs and smooth approximation with interactive Einstein operations, single-valued neutrosophic Einstein interactive weighted averaging and geometric operators are proposed. Based on proposed AOs, a robust MCDM approach is proposed for material selection in engineering design. A practical application of the proposed MCDM approach for material selection of cryogenic storage containers is developed. Additionally, the authenticity analysis and comparison analysis are designed to discuss the validity and rationality of the optimal decision.
\end{abstract}

Keywords Aggregation operators $\cdot$ Material selection $\cdot$ MCDM $\cdot$ Interaction relation $\cdot$ Einstein operational laws $\cdot$ Engineering design

\section{Introduction}

Problem resolution in our daily life is based on various stages like data collection, data analysis, information aggregation, etc. In decision-making analysis, the fundamental issue is the lack of accurate information. This information gap can be probably filled by employing mathematical modeling and adequate decision making procedure. The application of decision-making principles can help in the engineering design, the arrangement of various options, and the ranking of the feasible alternative from best to worst. As a result, it serves as a foundation for selecting, categorizing, and orga-

Hafiz Muhammad Athar Farid

hmatharfarid@gmail.com

Muhammad Riaz

mriaz.math@pu.edu.pk

1 Department of Mathematics, University of the Punjab, Lahore, Pakistan nizing our possibilities, as well as assisting in the overall assessment of the alternatives.

Material selection is critical in the design and development of products. The material chosen has an impact on the producers' success and competitiveness [1]. Engineering design is driven by the goals of performance, cost, and environmental sensitivity, and is generally constrained by materials. The purpose of optimum product design is to select materials that best fit the criteria of the design while providing maximum performance at the lowest possible cost [2]. However, in some competing scenarios, between these aims and parameters (e.g., cost, young modulus, hardness) are commonly observed, necessitating a decision on which feature is more significant than others. Material selection is an essential factor of the designing process money and resources can be squandered in the redesigning or fabrication of the planned part if the suitable material selection is not made during the designing process [3]. As there is no single right answer to a design issue, it is vital to choose the best material. To elimi- 
nate improper alternatives and determine the most acceptable one, the variables that influence material selection for a given engineering application must be evaluated using simple and logical procedures [4].

Manufacturing companies and engineers are constantly on the lookout for new materials and enhanced methods to utilize in the production of quality products, allowing them to keep their competitive edge and boost their gross profit [5]. Many outmoded materials that have long been used in engineering disciplines have been replaced in recent decades by so-called "new materials" to fulfil the requirement for performance increase, and weight reduction. The accessible collection of materials is continually expanding in both quantity and type [6]. There are more than 80,000 materials in the globe, according to estimates [7]. Nonmetallic and metallic alloy engineering materials, such as porcelain and glassware, as well as polymers, semiconductors, and compound materials, are all covered. Because of the huge number of materials available, as well as the intricate interactions between the various selection qualities, material selection for a given component is typically a strenuous task. Engineers and designers must consider a wide range of aspects when evaluating materials. These material factors include thermal and radiation (conductivity, specific heat, diffusivity, expansively, reflectivity, transmissivity, emissivity), electrical (permittivity, resistivity, dielectric strength), surface (texture, wear-resisting, corrosivity), manufacturing properties (machinability, castability, weldability, formability, heat treatability, etc.), physical properties ( crystal structure, vapor pressure, density, melting point, porosity, permeability, transparency, dimensional stability, optical properties), magnetic properties, mechanical properties (strength, Young's Modulus, elasticity, yield stress, creep resistance, fatigue, hardness and toughness, ductility), reliability, material cost, durability, fashion, material impact on the environment, recycle ability, aesthetics, market trends, availability, performance characteristics, cultural aspects, etc., [8-11]. Aside from these considerations, user-interaction features like appearance, perceptions, and emotions have lately been included in material selection. The customer interaction factors of a product are formulated as sensory material properties and have an impact on its usability and personality. Materials take on diverse meanings in different goods. According to Karana et al. [12] recent research, certain materials are associated with specific meanings such as professional, nostalgic, toy-like, aggressive, and attractive.

Automobile technological improvement has been implemented by enhancing its reliability and efficiency qualities. These modifications show that vehicle design, material requirements, and manufacturing methods all play an important impact. The initial step is to choose the optimum material for every single component [13]. Material selection is an aspect of sustainable development that relates to the establishment of materials that conserve resources, pursue a cleaner manufacturing environment, and are cost-effective [14]. The trial and error process necessitates the selection of other materials, which comes at a high cost; thus, the suitable instrument for material selection is critical.

MCDM approaches, which are used to handle conflicting concepts with numerous criteria, can be used to solve material selection problems with conflicting and non-commensurable criteria. In recent years, advancements in material selection have been made by taking environmental factors [15] into account, and the potential contribution of environmental impact study to environmental sustainability has been recognized and discussed [16]. Data aggregation is necessary for decision-making in the commercial, institutional, social, scientific, technological, artificial intelligence, and psychological areas. Traditionally, awareness of the alternative has been viewed as a linguistic term or crisp number. The data, however, cannot be effectively aggregated due to uncertainty. AOs have a significant role in the context of MCDM issues, the following principal goals of this manuscript are planned.

1. Single-valued neutrosophic numbers (SVNNs) are suitable to address the complexity issues practically with three components, indeterminacy, truthness, and falsity. Taking advantage of SVNSs, new aggregation operators (AOs) are proposed.

2. Einstein operators are well-known AOs for smooth approximation. It has been found that the existing Einstein AOs seem reluctant to make the right decision during the decision-making process in certain cases. To overcome these certain problems, these AOs must be modified. We present some innovative operations and their accompanying AOs for aggregating various SVNNs.

3. New aggregation operators are proposed named as "singlevalued neutrosophic Einstein interactive weighted averaging (SVNEIWA) operator", "single-valued neutrosophic Einstein interactive ordered weighted averaging (SVNEIOWA) operator", "single-valued neutrosophic Einstein interactive weighted geometric (SVNEIWG) operator" and "single-valued neutrosophic Einstein interactive ordered weighted geometric (SVNEIOWG) operator".

4. A sufficient number of illustrative examples are included to explain various innovative concepts related to newly AOs for information fusion. The suggested operators provide more generalized, reliable, and accurate information than previous techniques. With the help of suggested AOs, a new MCDM approach is developed for modeling uncertainty in real-life problems.

5. Material selection is an important facet of mechanical engineering since it meets all of the component's functional criteria. Material selection is a time-consuming but critical step in the design process. A manufacturer's out- 
put, profitability, and credibility can all suffer as a result of poor material(s) selection.

6. A cryogenic storage container is used as a case study to test the proposed method for material selection based on SVNSs. According to the findings, austenitic steel is the best material for the cryogenic storage container, which is consistent with real-world practise.

7. The authenticity analysis and comparison analysis of proposed MCDM approach with existing approaches is also presented to discuss the feasibility, authenticity, and superiority of the proposed method.

The remaining part of this article is organized as follows: contains a "Literature review". "Preliminaries" presents the ideas of SVNNs, their operational laws, and some basic Einstein aggregation operators for SVNNs. "SVN Einstein interactive aggregation "operators introduces the innovative concept of improved Einstein AOs for SVNNs. "Proposed approach for MCDM" presents a recommended approach for MCDM. "Case study" includes a case study, implementation of the suggested MCDM technique, authenticity analysis, and comparison analysis. The paper is concluded in "Conclusion".

\section{Literature review}

Materials selection is not a straightforward endeavor; thus, engineers and designers will benefit from the adoption of decision-support tools to assist them in making proper selections [17]. Inappropriate material selection frequently results in unfavorable economic consequences, and can eventually lead to premature product failure [18]. Different MCDM methods produce inconsistent results when selecting or evaluating a collection of alternative decisions incorporating several criteria [19]. Voogd demonstrated that each technique provided a different result than any other methodology at least $40 \%$ of the time [20]. This raises two critical concerns, both of which are difficult to answer: "How can a consensus ranking be reached when several distinct MCDM approaches rank differently?" and "Which technique is best suited to tackle the problem?" Several MCDM models have been developed with considerable effort, nonetheless, there is no ideal approach that is fundamentally superior to the others [21].

Shanian and Savadogo [22] presented the ELECTRE method for the material selection process but this technique has long and complicated calculations, and the computing procedure will grow complex as the number of alternatives expands. For material selection, Shanian and Savadogo [23] employed the TOPSIS technique; however, the methodology does not account for the qualitative different factors implicated in the judgment. Rao [24] selected materials using graph theory and a matrix technique. Because this strat- egy can take into account a wide range of quantitative and qualitative elements. However, there is no constraint in the approach for checking the consistency of the judgements of the relative importance of the criteria. Furthermore, if the number of traits exceeds 20, the approach may be difficult to use. Chan [25] proposed the MCDM method via grey relational analysis (GRA) to rate the material possibilities under non-linear restrictions, ambiguities, and conflicting goals. To rate the materials while taking environmental considerations into account, Chan and Tong [26] suggested a multi-criteria weighted average method based on grey relational analysis. When a single environmental score is used to select materials, Bovea and Gallardo [27] established the requirement for a sensitivity analysis.

Material qualities like wear and corrosion resistance, weldability, and machinability are rarely given numerical numbers, and materials are typically graded as poor, fair, very good, and so on. It appears that the ultimate material selection is jeopardized due to incomplete, estimated, and possibly inaccurate information. In such circumstances, fuzzy logic can be extremely beneficial. Fuzzy set theory was established on the concept that the essential factors in the human mind are linguistic phrases or fuzzy set labels rather than numbers. Because these judgments are made at the earliest design stages in an environment characterized by imprecise and uncertain requirements, parameters, and relationships, the use of fuzzy logic in material and process selection would also be beneficial [28]. Thurston and Carnahan [29] suggested using fuzzy analysis in the early stages of preliminary design review or in instances where design decision makers' input is limited to semantic information. Wang and Chang [30] have presented a fuzzy MCDM approach for the selection of tool steel materials. The material adequacy ratings of numerous alternatives under various criteria, as well as the importance weights of distinct criteria, were expressed in language terms. They described their fuzzy MCDM approach with a hypothetical case. Liao [31] presents a fuzzy MCDM method for material selection. Chen [32] suggested a method for aggregation and ranking that uses fundamental arithmetic laws rather than the complex arithmetic procedures disclosed by Wang and Chang [30]. Furthermore, Giachetti [28] combines a formal multi-attribute decision model with a relational database for material and manufacturing process selection. Because the values of material qualities are typically qualitatively expressed or imprecisely assessed using ranges, Giachetti employed possibility theory and the fuzzy set. His method's theoretical foundation was built on the evaluation of compatibility ratings between both the product profile specifications and each option. By Table 1, one can analyze the literature review regarding material selection.

It can be harder to find reliable assessment information due to the ambiguity in human subjective decisions. Because of the complexity in human subjective judgements, it may 


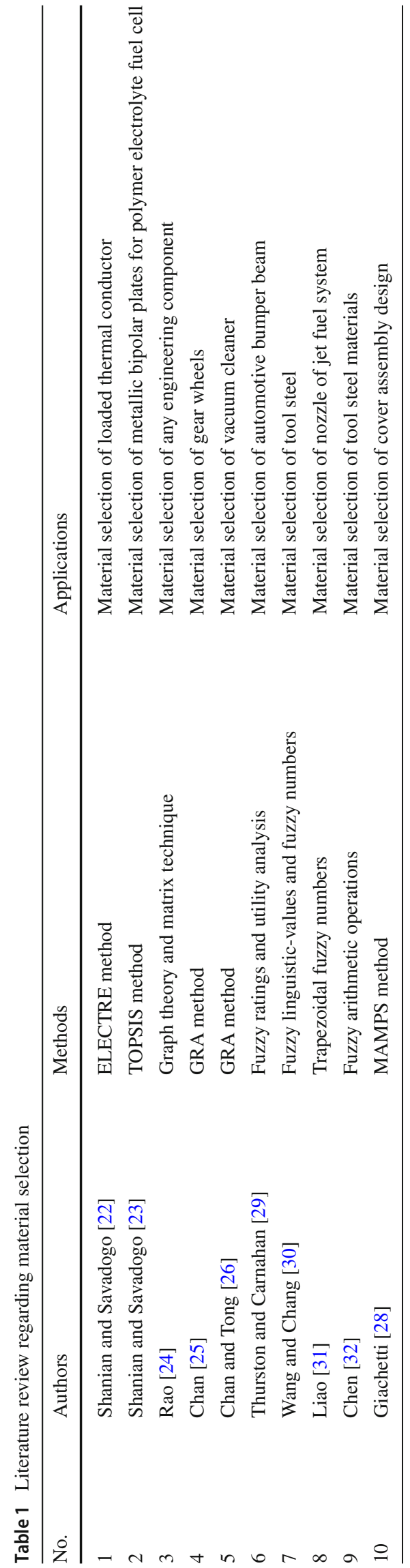

be more difficult to find valid assessment information. For decades, the issue of ambiguous and deceptive information has been a serious concern. Making decisions is one of the most fascinating aspects of our daily life. Although most assessments involve numerous measurements to reach their conclusion, some of them may be unclear. On the other hand, as the consequences of the structures grow by the day, it becomes increasingly difficult for the decision maker to make a reasonable judgement using unclear, erroneous, and imprecise facts in a reasonable period. MCDM is a conventional cognitive activity tool whose main goal is to choose between a limited number of possibilities based on preference information provided by DMs. However, the MCDM technique is unclear and imprecise since it incorporates the complexity of human reasoning abilities, making it difficult for DMs in the review process to provide an appropriate evaluation. It is essential to resolve this problem, in addition to dealing with unpredictability, Zadeh [33] has pioneered the sort of fuzzy set (FS) theory. Atanassov [34] initiated the novel idea of an intuitionistic fuzzy set (IFS). It has been pointed out that both the FS nor the IFS theories can deal with inconsistent and indeterminate data. Consider an expert who gives his or her opinion on a specific object, with 0.87 representing the likeliness that the assertion is correct 0.75 representing the likeliness that the assertion is incorrect and 0.29 representing the likeliness that he or she is unsure. To address this, Smarandache [35] proposed the idea of neutrosophic sets (NSs). In NS, each component in the universe of discourse set has varying degrees of truth membership degree (TMD), indeterminacy membership degree (IMD) and falsity membership degree (FMD) with values ranging from $\left(0^{-}, 1^{+}\right)$. Because of this non-standard unit interval, NS theory is difficult to apply to complex applications. So, in making it easier to be using NSs in applied mathematics, several classes of NSs and their interpretations were suggested. Wang et al. [36], presented class of single-valued NS (SVNS), Peng et al. [37] introduced simplified neutrosophic sets and Wang et al. [38] introduced Interval-valued Neutrosophic Sets. Because of its significance, a number of scientists have worked to improve the definition of NSs in the decision-making framework, using mechanisms including score functions [39], distance measures [40] and others.

Traditionally, awareness of the alternative has been viewed as a crisp number or linguistic number. The data, however, cannot be effectively aggregated due to its uncertainty. In fact, AOs play an important role in the context of MCDM issues, the main goal of which is to aggregate a series of inputs to a single number. Ye [41] introduced the operational laws of SVNSs and suggested the averaging and geometric AOs for SVNNs. Peng et al. [42] proposed upgraded SVNN operations and established their associated AOs. Nancy and Garg [43] established AOs by employing Frank operations. Liu et al. [44] created some AOs for SVNNs based on Hamacher 
operations. Zhang et al. [45] provided the AOs in the context of an interval-valued neutrosophic set. Wu et al. [46] introduced prioritized AOs with Simplified Neutrosophic Sets. Li et al. [47] presented the novel idea of generalized simplified neutrosophic Einstein AOs. Wei and Wei [48] developed dombi prioritized AOs for SVNSs. Liu [49] gave the idea of AOs based on archimedean t-norm and t-conorm for SVNSs. Garg and Nancy [50] gave the novel idea of prioritized muirhead mean AOs under NSs. Wang et al. [51] explored the dual BM aggregation operator for SVNNs further. Harmonic mean AOs for SVNNs were presented by Mondal et al. [52]. Ji et al. [53] demonstrated the Frank prioritized BM in an SVN environment by their application in third-party logistics selection. Wei and Zhang [54] utilized single-valued neutrosophic Bonferroni power AOs to choose strategic providers.

Yang et al. [55] presented continuous ordered weighted averaging AOs for interval-valued q-ROF information and their use in quality assessment of smartwatch aesthetic design. Chen et al. [56] introduced the notion of enhanced ordered weighted averaging AOs and their application towards MCDM. Chen et al. [57] also proposed Poweraverage AOs for a proportional hesitant fuzzy linguistic term set, with an implementation to an online product recommender system for consumer decision-making. Chen et al. [58] proposed large-scale group decision-making for determining passenger demands and evaluating passenger satisfaction. Chen et al. [59] presented the idea of linguistic ELECTRE III and their application to constructioncontractor selection. The concept of linear Diophantine fuzzy Set (LDFS) was introduced by Riaz and Hashmi [60]. Riaz et al. introduced Einstein AOs [62] and prioritized AOs [61] related to LDFSs. Liu and Liu [63] initiated the idea of q-ROF Bonferroni mean AOs. Riaz et al. [64] introduced the novel concept of bipolar picture fuzzy. Liu et al. [65] initiated the idea of q-ROF Heronianmean AOs and application related to MCDM. . Riaz et al. presented a holistic approach towards q-ROF interactive AOs [66] and AOs related to q-ROF soft set [67]. Ye et al. [68] introduced MCDM method based on fuzzy rough sets. Mu et al. [69] developed power Maclaurin symmetric mean AOs based on interval-valued Pythagorean fuzzy set.

\section{Preliminaries}

Some fundamental concepts related to SVNSs have been presented in this section, over the universal set $\Theta$.

Definition 3.1 [36] A single-valued neutrosophic set (SVNS) a in $\Theta$ is defined as

$\chi=\left\{\left\langle x, \mu_{\chi}^{\epsilon}(x), \lambda_{\chi}(x), v_{\chi}(x) \mid x \in \Theta\right\rangle\right\}$,

where $\mu^{\epsilon}{ }_{\chi}(x), \lambda_{\chi}(x), v_{\chi}(x) \in[0,1]$, such that $0 \leq$ $\mu^{\epsilon}{ }_{\chi}(x)+\lambda_{\chi}(x)+v_{\chi}(x) \leq 3$ for all $x \in \Theta . \mu^{\epsilon}{ }_{\chi}(x), \lambda_{\chi}(x)$, $v_{\chi}(x)$ denote TMD, IMD and FMD, respectively, for some $x \in \Theta$.

We denote this pair as $\alpha=\left(\mu_{\alpha}^{\epsilon}, \lambda_{\alpha}, v_{\alpha}\right)$, throughout this article, and called as SVNN with the conditions $\mu_{\alpha}^{\epsilon}, \lambda_{\alpha}, v_{\alpha} \in[0,1]$ and $\mu_{\alpha}^{\epsilon}+\lambda_{\alpha}+v_{\alpha} \leq 3$.

Now, we will present some operational rules to aggregate the SVNNs.

Definition $3.2[36,37]$ Let $\alpha_{1}=\left\langle\mu^{\epsilon}{ }_{1}, \lambda_{1}, \nu_{1}\right\rangle$ and $\alpha_{2}=$ $\left\langle\mu_{2}^{\epsilon}, \lambda_{2}, v_{2}\right\rangle$ be to SVNNs, then

$\alpha_{1}^{c}=\left\langle v_{1}, \lambda_{1}, \mu^{\epsilon}{ }_{1}\right\rangle$,

$\alpha_{1} \vee \alpha_{2}=\left\langle\max \left\{\mu^{\epsilon}{ }_{1}, \mu^{\epsilon}{ }_{2}\right\}, \min \left\{\lambda_{1}, \lambda_{2}\right\}, \min \left\{v_{1}, \nu_{2}\right\}\right\rangle$,

$\alpha_{1} \wedge \alpha_{2}=\left\langle\min \left\{\mu^{\epsilon}{ }_{1}, \mu^{\epsilon}{ }_{2}\right\}, \max \left\{\lambda_{1}, \lambda_{2}\right\}, \max \left\{\nu_{1}, \nu_{2}\right\}\right\rangle$,

$\alpha_{1} \oplus \alpha_{2}=\left\langle\mu^{\epsilon}{ }_{1}+\mu^{\epsilon}{ }_{2}-\mu^{\epsilon}{ }_{1} \mu^{\epsilon}{ }_{2}, \lambda_{1} \lambda_{2}, v_{1} \nu_{2}\right\rangle$,

$\alpha_{1} \otimes \alpha_{2}=\left\langle\mu^{\epsilon}{ }_{1} \mu_{2}^{\epsilon}, \lambda_{1}+\lambda_{2}-\lambda_{1} \lambda_{2}, \nu_{1}+\nu_{2}-v_{1} \nu_{2}\right\rangle$,

$\sigma \alpha_{1}=\left\langle 1-\left(1-\mu_{1}^{\epsilon}\right)^{\sigma}, \lambda_{1}^{\sigma}, v_{1}^{\sigma}\right\rangle$,

$\alpha_{1}^{\sigma}=\left\langle\mu_{1}^{\epsilon \sigma}, 1-\left(1-\lambda_{1}\right)^{\sigma}, 1-\left(1-v_{1}\right)^{\sigma}\right\rangle$.

Definition 3.3 [46] Let $\alpha=\left\langle\mu^{\epsilon}{ }_{\alpha}, \lambda_{\alpha}, v_{\alpha}\right\rangle$ be the SVNN, then the score function can be defined as follows.

$\breve{\Xi}(\alpha)=\frac{\mu_{\alpha}^{\epsilon}+1-\lambda_{\alpha}+1-v_{\alpha}}{3}$.

If we have two SVNNs say $\alpha$ and $\beta$ if $\breve{\Xi}(\alpha)>\breve{\Xi}(\beta)$, then $\alpha>\beta$.

\section{SVN Einstein operations}

For SVNNs, Li et al. presented the Einstein operation and investigated its attractive properties.

Definition $3.4[47] \alpha_{1}=\left\langle\mu^{\epsilon}{ }_{1}, \lambda_{1}, \nu_{1}\right\rangle$ and $\alpha_{2}=\left\langle\mu^{\epsilon}{ }_{2}, \lambda_{2}, \nu_{2}\right\rangle$ be to SVNNs, then $\breve{\wp}>0$ be real number, then

$$
\begin{gathered}
\alpha_{1} \otimes_{\epsilon} \alpha_{2}=\left\langle\frac{\mu_{1 \cdot \epsilon}^{\epsilon} \mu_{2}^{\epsilon}}{1+\left(1-\mu^{\epsilon}\right)_{1 \epsilon}\left(1-\mu^{\epsilon}\right)}, \frac{\lambda_{1}+\lambda_{2}}{1+\lambda_{1 \cdot \epsilon} \lambda_{2}},\right. \\
\left.\frac{v_{1}+\nu_{2}}{1+v_{1 \cdot \epsilon} \nu_{2}}\right\rangle,
\end{gathered}
$$

$\alpha_{1} \oplus_{\epsilon} \alpha_{2}=\left\langle\frac{\mu^{\epsilon}{ }_{1}+\mu^{\epsilon}{ }_{2}}{1+\mu^{\epsilon}{ }_{1 \cdot \epsilon} \mu^{\epsilon}}, \frac{\lambda_{1 \cdot \epsilon} \lambda_{2}}{1+\left(1-\lambda_{1}\right)_{\cdot \epsilon}\left(1-\lambda_{2}\right)}\right.$,

$$
\left.\frac{v_{1 \cdot \epsilon} \nu_{2}}{1+\left(1-v_{1}\right) \cdot \epsilon\left(1-v_{2}\right)}\right\rangle \text {, }
$$

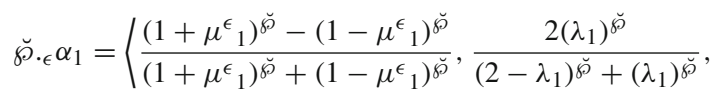

$$
\left.\frac{2\left(v_{1}\right)^{\breve{\varphi}}}{\left(2-v_{1}\right)^{\check{\wp}}+\left(v_{1}\right)^{\breve{\varphi}}}\right\rangle \text {, }
$$




$$
\begin{aligned}
& \alpha_{1}^{\breve{\wp}}=\left\langle\frac{2\left(\mu^{\epsilon}{ }_{1}\right)^{\breve{\wp}}}{\left(2-\mu^{\epsilon}{ }_{1}\right)^{\breve{9}}+\left(\mu^{\epsilon}{ }_{1}\right)^{\breve{\wp}}}, \frac{\left(1+\left(\lambda_{1}\right)^{\breve{\wp}}-\left(1-\lambda_{1}\right)^{\breve{\wp}}\right.}{\left(1+\lambda_{1}\right)^{\breve{\varphi}}+\left(1-\lambda_{1}\right)^{\breve{\wp}}},\right. \\
& \frac{\left(1+\left(v_{1}\right)^{\breve{\varphi}}-\left(1-v_{1}\right)^{\breve{\varphi}}\right.}{\left(1+v_{1}\right)^{\breve{\varphi}}+\left(1-v_{1}\right)^{\breve{\wp}}} \text {. }
\end{aligned}
$$

Based on these operations, Li et al. [47] introduced some SVN Einstein averaging AOs.

Definition 3.5 Let $\alpha_{p}=\left\langle\mu^{\epsilon}{ }_{p}, \lambda_{p}, v_{p}\right\rangle$ be the assemblage of SVNNs. Then

$$
\begin{aligned}
& \operatorname{SVNEWA}\left(\alpha_{1}, \alpha_{2}, \ldots, \alpha_{n}\right) \\
& =\left(\frac{\prod_{s=1}^{n}\left(1+\mu^{\epsilon}{ }_{s}\right)^{\omega_{s}}-\prod_{s=1}^{n}\left(1-\mu^{\epsilon}{ }_{s}\right)^{\omega_{s}}}{\prod_{s=1}^{n}\left(1+\mu^{\epsilon}{ }_{s}\right)^{\omega_{s}}+\prod_{s=1}^{n}\left(1-\mu^{\epsilon}\right)^{\omega_{s}}},\right. \\
& \\
& \quad \frac{2 \prod_{s=1}^{n} \lambda_{s}^{\omega_{s}}}{\prod_{s=1}^{n}\left(2-\lambda_{s}\right)^{\omega_{s}}+\prod_{s=1}^{n}\left(\lambda_{s}\right)^{\omega_{s}}} \\
& \left.\quad \frac{2 \prod_{s=1}^{n} v_{s}^{\omega_{s}}}{\prod_{s=1}^{n}\left(2-v_{s}\right)^{\omega_{s}}+\prod_{s=1}^{n}\left(v_{s}\right)^{\omega_{s}}}\right),
\end{aligned}
$$

where $\omega=\left(\omega_{1}, \omega_{2}, \ldots, \omega_{n}\right)^{T}$ is the weight vector $(\mathrm{WV})$ of $\left(\alpha_{1}, \alpha_{2}, \ldots, \alpha_{n}\right)$, such that $0 \leq \omega_{s} \leq 1$ and $\sum_{s=1}^{n} \omega_{s}=1$. Then, the SVNEWA is called the single-valued neutrosophic Einstein weighted averaging operator.

Definition 3.6 Let $\alpha_{p}=\left\langle\mu^{\epsilon}{ }_{p}, \lambda_{p}, v_{p}\right\rangle$ be the assemblage of SVNNs, we can also find SVNEWA by

$$
\begin{aligned}
& \operatorname{SVNEOWA}\left(\alpha_{1}, \alpha_{2}, \ldots, \alpha_{n}\right) \\
& =\left(\frac{\prod_{s=1}^{n}\left(1+\mu_{£(s)}^{\epsilon}\right)^{\omega_{s}}-\prod_{s=1}^{n}\left(1-\mu_{£(s)}^{\epsilon}\right)^{\omega_{s}}}{\prod_{s=1}^{n}\left(1+\mu_{£(s)}^{\epsilon}\right)^{\omega_{s}}+\prod_{s=1}^{n}\left(1-\mu_{£(s)}^{\epsilon}\right)^{\omega_{s}}},\right. \\
& \frac{2 \prod_{s=1}^{n} \lambda_{£(s)}^{\omega_{s}}}{\prod_{s=1}^{n}\left(2-\lambda_{£(s)}\right)^{\omega_{s}}+\prod_{s=1}^{n}\left(\lambda_{£(s)}\right)^{\omega_{s}}}, \\
& \left.\frac{2 \prod_{s=1}^{n} v_{£(s)}^{\omega_{s}}}{\prod_{s=1}^{n}\left(2-v_{£(s)}\right)^{\omega_{s}}+\prod_{s=1}^{n}\left(v_{£(s)}\right)^{\omega_{s}}}\right),
\end{aligned}
$$

where $\omega=\left(\omega_{1}, \omega_{2}, \ldots, \omega_{n}\right)^{T}$ is the $\mathrm{WV}$ of $\left(\alpha_{1}, \alpha_{2}, \ldots, \alpha_{n}\right)$, such that $0 \leq \omega_{s} \leq 1$ and $\sum_{s=1}^{n} \omega_{s}=1 . £(1), £(2), \ldots, £(n)$ is a permutation of $(1,2, \ldots, n)$ such that $\alpha_{\mathfrak{f}(i-1)} \geq \alpha_{\mathfrak{f}(i)}$. Then, the SVNEOWA is called the single valued neutrosophic Einstein ordered weighted averaging operator.

\section{Insufficiencies of the existing SVN Einstein averaging $\mathrm{AOs}$}

It has been found that the above-mentioned AOs seem reluctant to make the right decision during the decision-making process in certain cases. The following explanations demonstrate this point.

- During the aggregation process, there is no impact of TMDs on IMDs and FMDs. We found that by modifying the degrees of the TMDs of the SVNNs in the
SVNEWA operator, the collective IMDs and FMDs of the SVNEWA operator remain independently of its. The following example demonstrates this.

Example 3.7 Assume an assemblage of three SVNNs $\alpha_{1}=$ $\langle 0.75,0.75,0.00\rangle, \alpha_{2}=\langle 0.35,0.00,0.45\rangle$ and $\alpha_{3}=$ $\langle 0.55,0.35,0.15\rangle$ with $\mathrm{WV} \omega=(0.30,0.30,0.40)^{T}$. Then, using the SVNEWA operator, we can aggregate these numbers to get $\operatorname{SVNEWA}\left(\alpha_{1}, \alpha_{2}, \alpha_{3}\right)=\langle 0.57091,0.00000$, $0.00000\rangle$. If we modify the TMDs of the above SVNNs, we will get a diverse assortment, $\beta_{1}=\langle 0.85,0.75,0.00\rangle, \beta_{2}=$ $\langle 0.25,0.00,0.45\rangle$ and $\beta_{3}=\langle 0.95,0.35,0.15\rangle$ with the same $\mathrm{WV}$, then by SVNEWA operator, we get aggregated SVNN corresponding to these $\operatorname{SVNNs}$ is $\operatorname{SVNEWA}\left(\beta_{1}, \beta_{2}, \beta_{3}\right)=$ $\langle 0.829391,0.00000,0.00000\rangle$

As a result, we indicate that modifying the grading of TMD has no effect on IMDs and FMDs.

- Non-zero IMDs and FMDs values have no impact on the outcome of an AOs. According to the formulation of SVNEWA, if at least one of the degree of IMDs and FMDs is zero, the aggregate IMD and FMD of the SVNEWA operator becomes zero, respectively. This has been demonstrated with a numerical example, which can be found in Example 3.7. It can be seen from this that the IMD and FMD of the SVNEWA operator become zero as a result of the zero FMD of $\alpha_{1}$ and IMD of $\alpha_{2}$. As a result, the present operator SVNEWA usually produces an erroneous result when ranking the alternatives.

To address the aforementioned problem, these AOs must be modified. In the following section, we suggest some innovative operations and their accompanying AOs for aggregating various SVNNs.

\section{SVN Einstein interactive aggregation operators}

In this section, we will initially interpret some improved Einstein operations of the SVNNs that consider the interaction of TMDs, IMDs and FMDs. Then, we define some SVN Einstein interactive averaging AO.

\section{Improved Einstein operational laws on SVNNs}

Definition 4.1 Let $\alpha_{1}=\left\langle\mu^{\epsilon}{ }_{1}, \lambda_{1}, \nu_{1}\right\rangle, \alpha_{2}=\left\langle\mu^{\epsilon}{ }_{2}, \lambda_{2}, \nu_{2}\right\rangle$ and $\alpha=\left\langle\mu^{\epsilon}, \lambda, \nu\right\rangle$ be SVNNs and $\breve{\wp}>0$ be any real number. The Einstein norm operations between them have been defined as 


$$
\begin{aligned}
\alpha_{1} \oplus \alpha_{2}= & \frac{\prod_{s=1}^{2}\left(1+\mu^{\epsilon}\right)-\prod_{s=1}^{2}\left(1-\mu_{s}^{\epsilon}\right)}{\prod_{s=1}^{2}\left(1+\mu^{\epsilon}\right)+\prod_{s=1}^{2}\left(1-\mu_{s}^{\epsilon}\right)}, \\
& \frac{\prod_{s=1}^{2}\left(1+\mu^{\epsilon}{ }_{s}-\lambda_{s}\right)}{2\left(\prod_{s=1}^{2}\left(1+\mu^{\epsilon}\right)+\prod_{s=1}^{2}\left(1-\mu_{s}^{\epsilon}\right)\right)}, \\
& \left.\frac{\prod_{s=1}^{2}\left(1+\mu_{s}^{\epsilon}-v_{s}\right)}{2\left(\prod_{s=1}^{2}\left(1+\mu^{\epsilon}\right)+\prod_{s=1}^{2}\left(1-\mu_{s}^{\epsilon}\right)\right)}\right),
\end{aligned}
$$

$\alpha_{1} \otimes \alpha_{2}=\left(\frac{\prod_{s=1}^{2}\left(1+v_{s}-\mu^{\epsilon}\right)}{2\left(\prod_{s=1}^{2}\left(1+v_{s}\right)+\prod_{s=1}^{2}\left(1-v_{s}\right)\right)}\right.$,

$$
\begin{aligned}
& \frac{\prod_{s=1}^{2}\left(1+\lambda_{s}\right)-\prod_{s=1}^{2}\left(1-\lambda_{s}\right)}{\prod_{s=1}^{2}\left(1+\lambda_{s}\right)+\prod_{s=1}^{2}\left(1-\lambda_{s}\right)}, \\
& \left.\frac{\prod_{s=1}^{2}\left(1+v_{s}\right)-\prod_{s=1}^{2}\left(1-v_{s}\right)}{\prod_{s=1}^{2}\left(1+v_{s}\right)+\prod_{s=1}^{2}\left(1-v_{s}\right)}\right),
\end{aligned}
$$

$$
\breve{\wp} \alpha=\left(\frac{\left(1+\mu^{\epsilon}\right)^{\breve{\wp}}-\left(1-\mu^{\epsilon}\right)^{\breve{\wp}}}{\left(1+\mu^{\epsilon}\right)^{\breve{\wp}}+\left(1-\mu^{\epsilon}\right)^{\breve{\wp}}}, \frac{\left(1+\mu^{\epsilon}-\lambda\right)^{\breve{\wp}}}{2\left(\left(1+\mu^{\epsilon}\right)^{\breve{\wp}}+\left(1-\mu^{\epsilon}\right)^{\breve{\wp}}\right)}\right. \text {, }
$$

$$
\begin{aligned}
& \left.\frac{\left(1+\mu^{\epsilon}-v\right)^{\breve{\wp}}}{2\left(\left(1+\mu^{\epsilon}\right)^{\breve{\wp}}+\left(1-\mu^{\epsilon}\right)^{\breve{\wp}}\right)}\right) \text {, } \\
& \alpha^{\breve{\wp}}=\left(\frac{\left(1+v-\mu^{\epsilon}\right)^{\breve{\wp}}}{2\left((1+v)^{\breve{\wp}}+(1-v)^{\breve{\wp}}\right)}, \frac{(1+\lambda)^{\breve{\varphi}}-(1-\lambda)^{\breve{\wp}}}{(1+\lambda)^{\breve{\varphi}}+(1-\lambda)^{\breve{\wp}}},\right. \\
& \left.\frac{(1+v)^{\breve{\varphi}}-(1-v)^{\breve{\varphi}}}{(1+v)^{\breve{\varphi}}+(1-v)^{\breve{\varphi}}}\right) \text {. }
\end{aligned}
$$

\section{SVNEIWA operator}

We will examine the weighted averaging AOs based on these improved Einstein laws in the following manner.

Definition 4.2 Let $\alpha_{p}=\left\langle\mu^{\epsilon}{ }_{p}, \lambda_{p}, v_{p}\right\rangle$ be the assemblage of SVNNs and (SVNEIWA): $\Lambda^{n} \rightarrow \Lambda$ if,

$$
\begin{aligned}
\operatorname{SVNEIWA}\left(\alpha_{1}, \alpha_{2}, \ldots, \alpha_{n}\right) & =\bigoplus_{s=1}^{n} \omega_{s} \alpha_{s} \\
& =\omega_{1} \alpha_{1} \oplus \omega_{2} \alpha_{2} \oplus \ldots \oplus \omega_{n} \alpha_{n}
\end{aligned}
$$

$\omega=\left(\omega_{1}, \omega_{2}, \ldots, \omega_{n}\right)^{T}$ is the WV of $\left(\alpha_{1}, \alpha_{2}, \ldots, \alpha_{n}\right)$, such that $0 \leq \omega_{s} \leq 1$ and $\sum_{s=1}^{n} \omega_{s}=1$ and where $\Lambda$ is the set of all SVNNs. Then, the SVNEIWA is called the singlevalued neutrosophic Einstein interactive weighted averaging (SVNEIWA) operator.

Theorem 4.3 Let $\alpha_{p}=\left\langle\mu_{p}^{\epsilon}, \lambda_{p}, v_{p}\right\rangle$ be the assemblage of SVNNs, then

$$
\begin{aligned}
& \operatorname{SVNEIWA}\left(\alpha_{1}, \alpha_{2}, \ldots, \alpha_{n}\right) \\
& =\frac{\left(\frac{\prod_{s=1}^{n}\left(1+\mu^{\epsilon}{ }_{s}\right)^{\omega_{s}}-\prod_{s=1}^{n}\left(1-\mu^{\epsilon}{ }_{s}\right)^{\omega_{s}}}{\prod_{s=1}^{n}\left(1+\mu^{\epsilon}\right)^{\omega_{s}}+\prod_{s=1}^{n}\left(1-\mu^{\epsilon}{ }_{s}\right)^{\omega_{s}}},\right.}{2\left(\prod_{s=1}^{n}\left(1+\mu^{\epsilon}{ }_{s}\right)^{\omega_{s}}+\prod_{s=1}^{n}\left(1-\mu^{\epsilon}{ }_{s}\right)^{\omega_{s}}\right)}, \\
& \left.\frac{\prod_{s=1}^{n}\left(1+\mu^{\epsilon}{ }_{s}-v_{s}\right)^{\omega_{s}}\left(1+\mu^{\epsilon}-\lambda_{s}\right)^{\omega_{s}}}{2\left(\prod_{s=1}^{n}\left(1+\mu^{\epsilon}\right)^{\omega_{s}}+\prod_{s=1}^{n}\left(1-\mu^{\epsilon}{ }_{s}\right)^{\omega_{s}}\right)}\right) .
\end{aligned}
$$

Proof We will prove this theorem by mathematical induction. When $n=1, \omega=1$, we have

$$
\begin{aligned}
& \text { SVNEIWA }\left(\alpha_{1}\right)=\omega_{1} \alpha_{1} \\
& =\left(\frac{\left(1+\mu^{\epsilon}{ }_{1}\right)^{1}-\left(1-\mu^{\epsilon}{ }_{1}\right)^{1}}{\left(1+\mu^{\epsilon}\right)^{1}+\left(1-\mu^{\epsilon}\right)^{1}}, \frac{\left(1+\mu^{\epsilon}{ }_{1}-\lambda_{1}\right)^{1}}{2\left(\left(1+\mu^{\epsilon}\right)_{1}^{1}+\left(1-\mu^{\epsilon}\right)^{1}\right)},\right. \\
& \\
& \left.\frac{\left(1+\mu^{\epsilon}{ }_{1}-v_{1}\right)^{1}}{2\left(\left(1+\mu^{\epsilon}\right)^{1}+\left(1-\mu^{\epsilon}\right)^{1}\right)}\right) .
\end{aligned}
$$

Thus, theorem holds for $n=1$.

Now, we have for $n=2$

$$
\begin{aligned}
& \omega_{1} \alpha_{1}=\left(\frac{\left(1+\mu^{\epsilon}{ }_{1}\right)^{\omega_{1}}-\left(1-\mu_{1}^{\epsilon}\right)^{\omega_{1}}}{\left(1+\mu^{\epsilon}{ }_{1}\right)^{\omega_{1}}+\left(1-\mu^{\epsilon}\right)^{\omega_{1}}},\right. \\
& \frac{\left(1+\mu^{\epsilon}{ }_{1}-\lambda_{1}\right)^{\omega_{1}}}{2\left(\left(1+\mu^{\epsilon}\right)^{\omega_{1}}+\left(1-\mu^{\epsilon}\right)^{\omega_{1}}\right)}, \\
& \left.\frac{\left(1+\mu^{\epsilon}{ }_{1}-v_{1}\right)^{\omega_{1}}}{2\left(\left(1+\mu^{\epsilon}\right)^{\omega_{1}}+\left(1-\mu^{\epsilon}{ }_{1}\right)^{\omega_{1}}\right)}\right) \\
& =\left(\frac{\tau_{1}-\tau_{2}}{\tau_{1}+\tau_{2}}, \frac{\Omega_{3}}{2\left(\tau_{1}+\tau_{2}\right)}, \frac{\tau_{3}}{2\left(\tau_{1}+\tau_{2}\right)}\right), \\
& \omega_{2} \alpha_{2}=\left(\frac{\left(1+\mu^{\epsilon}{ }_{2}\right)^{\omega_{2}}-\left(1-\mu_{2}^{\epsilon}\right)^{\omega_{2}}}{\left(1+\mu^{\epsilon}\right)^{\omega_{2}}+\left(1-\mu^{\epsilon}\right)^{\omega_{2}}},\right. \\
& \frac{\left(1+\mu^{\epsilon}{ }_{2}-\lambda_{2}\right)^{\omega_{2}}}{2\left(\left(1+\mu^{\epsilon}{ }_{2}\right)^{\omega_{2}}+\left(1-\mu^{\epsilon}{ }_{2}\right)^{\omega_{2}}\right)}, \\
& \left.\frac{\left(1+\mu_{2}^{\epsilon}-v_{2}\right)^{\omega_{2}}}{2\left(\left(1+\mu^{\epsilon}{ }_{2}\right)^{\omega_{2}}+\left(1-\mu^{\epsilon}\right)^{\omega_{2}}\right)}\right) \\
& =\left(\frac{\Xi_{1}-\Xi_{2}}{\Xi_{1}+\Xi_{2}}, \frac{\Phi_{3}}{2\left(\Xi_{1}+\Xi_{2}\right)}, \frac{\Xi_{3}}{2\left(\Xi_{1}+\Xi_{2}\right)}\right) \text {, }
\end{aligned}
$$

where $\Xi_{1}=\left(1+\mu_{2}^{\epsilon}\right)^{\omega_{2}}, \quad \Xi_{2}=\left(1-\mu_{2}^{\epsilon}\right)^{\omega_{2}}$, $\Xi_{3}=\left(1+\mu_{2}^{\epsilon}-\nu_{2}\right)^{\omega_{2}}, \quad \Phi_{3}=\left(1+\mu_{2}^{\epsilon}-\lambda_{2}\right)^{\omega_{2}}$,

$\tau_{1}=\left(1+\mu_{1}^{\epsilon}\right)^{\omega_{1}}, \quad \tau_{2}=\left(1-\mu^{\epsilon}{ }_{1}\right)^{\omega_{1}}, \quad \tau_{3}=(1+$ $\left.\mu_{1}^{\epsilon}-v_{1}\right)^{\omega_{1}}, \quad \Omega_{3}=\left(1+\mu^{\epsilon}{ }_{1}-\lambda_{1}\right)^{\omega_{1}}$. 
$\operatorname{SVNEIWA}\left(\alpha_{1}, \alpha_{2}\right)=\omega_{1} \alpha_{1} \oplus \omega_{2} \alpha_{2}$

$$
\begin{aligned}
& =\left(\frac{\left(1+\mu^{\epsilon}{ }_{1}\right)^{\omega_{1}}-\left(1-\mu_{1}^{\epsilon}\right)^{\omega_{1}}}{\left(1+\mu^{\epsilon}\right)^{\omega_{1}}+\left(1-\mu^{\epsilon}\right)^{\omega_{1}}}, \frac{\left(1+\mu^{\epsilon}{ }_{1}-\lambda_{1}\right)^{\omega_{1}}}{2\left(\left(1+\mu^{\epsilon}\right)^{\omega_{1}}+\left(1-\mu^{\epsilon}\right)^{\omega_{1}}\right)}, \frac{\left(1+\mu^{\epsilon}{ }_{1}-v_{1}\right)^{\omega_{1}}}{2\left(\left(1+\mu^{\epsilon}\right)^{\omega_{1}}+\left(1-\mu^{\epsilon}\right)^{\omega_{1}}\right)}\right) \\
& \oplus\left(\frac{\left(1+\mu_{2}^{\epsilon}\right)^{\omega_{2}}-\left(1-\mu_{2}^{\epsilon}\right)^{\omega_{2}}}{\left(1+\mu^{\epsilon}{ }^{\omega_{2}}+\left(1-\mu^{\epsilon}\right)^{\omega_{2}}\right.}, \frac{\left(1+\mu^{\epsilon}{ }_{2}-\lambda_{2}\right)^{\omega_{2}}}{2\left(\left(1+\mu^{\epsilon}\right)^{\omega_{2}}+\left(1-\mu^{\epsilon}{ }_{2}\right)^{\omega_{2}}\right)}, \frac{\left(1+\mu^{\epsilon}{ }_{2}-v_{2}\right)^{\omega_{2}}}{2\left(\left(1+\mu^{\epsilon}{ }_{2}\right)^{\omega_{2}}+\left(1-\mu^{\epsilon}\right)^{\omega_{2}}\right)}\right) \\
& =\left(\frac{\tau_{1}-\tau_{2}}{\tau_{1}+\tau_{2}}, \frac{\Omega_{3}}{2\left(\tau_{1}+\tau_{2}\right)}, \frac{\tau_{3}}{2\left(\tau_{1}+\tau_{2}\right)}\right) \oplus\left(\frac{\Xi_{1}-\Xi_{2}}{\Xi_{1}+\Xi_{2}}, \frac{\Phi_{3}}{2\left(\Xi_{1}+\Xi_{2}\right)}, \frac{\Xi_{3}}{2\left(\Xi_{1}+\Xi_{2}\right)}\right) \\
& =\left(\frac{\left(1+\frac{\left(\tau_{1}-\tau_{2}\right)}{\tau_{1}+\tau_{2}}\right)\left(1+\frac{\Xi_{1}-\Xi_{2}}{\Xi_{1}+\Xi_{2}}\right)-\left(1-\frac{\left(\tau_{1}-\tau_{2}\right)}{\tau_{1}+\tau_{2}}\right)\left(1-\frac{\Xi_{1}-\Xi_{2}}{\Xi_{1}+\Xi_{2}}\right)}{\left(1+\frac{\left(\tau_{1}-\tau_{2}\right)}{\tau_{1}+\tau_{2}}\right)\left(1+\frac{\Xi_{1}-\Xi_{2}}{\Xi_{1}+\Xi_{2}}\right)+\left(1-\frac{\left(\tau_{1}-\tau_{2}\right)}{\tau_{1}+\tau_{2}}\right)\left(1-\frac{\Xi_{1}-\Xi_{2}}{\Xi_{1}+\Xi_{2}}\right)},\right. \\
& \left(1+\frac{\left(\tau_{1}-\tau_{2}\right)}{\tau_{1}+\tau_{2}}-\frac{\Omega_{3}}{2\left(\tau_{1}+\tau_{2}\right)}\right)\left(1+\frac{\left(\Xi_{1}-\Xi_{2}\right)}{\Xi_{1}+\Xi_{2}}-\frac{\Phi_{3}}{2\left(\Xi_{1}+\Xi_{2}\right)}\right) \\
& \overline{2\left(\left(1+\frac{\left(\tau_{1}-\tau_{2}\right)}{\tau_{1}+\tau_{2}}\right)\left(1+\frac{\Xi_{1}-\Xi_{2}}{\Xi_{1}+\Xi_{2}}\right)+\left(1-\frac{\left(\tau_{1}-\tau_{2}\right)}{\tau_{1}+\tau_{2}}\right)\left(1-\frac{\Xi_{1}-\Xi_{2}}{\Xi_{1}+\Xi_{2}}\right)\right)}, \\
& \left.\frac{\left(1+\frac{\left(\tau_{1}-\tau_{2}\right)}{\tau_{1}+\tau_{2}}-\frac{\tau_{3}}{2\left(\tau_{1}+\tau_{2}\right)}\right)\left(1+\frac{\left(\Xi_{1}-\Xi_{2}\right)}{\Xi_{1}+\Xi_{2}}-\frac{\Xi_{3}}{2\left(\Xi_{1}+\Xi_{2}\right)}\right)}{2\left(\left(1+\frac{\left(\tau_{1}-\tau_{2}\right)}{\tau_{1}+\tau_{2}}\right)\left(1+\frac{\Xi_{1}-\Xi_{2}}{\Xi_{1}+\Xi_{2}}\right)+\left(1-\frac{\left(\tau_{1}-\tau_{2}\right)}{\tau_{1}+\tau_{2}}\right)\left(1-\frac{\Xi_{1}-\Xi_{2}}{\Xi_{1}+\Xi_{2}}\right)\right)}\right) \\
& =\left(\frac{\tau_{1} \Xi_{1}-\tau_{2} \Xi_{2}}{\tau_{1} \Xi_{1}+\tau_{2} \Xi_{2}}, \frac{\Omega_{3} \Phi_{3}}{2\left(\tau_{1} \Xi_{1}+\tau_{2} \Xi_{2}\right)} \frac{\tau_{3} \Xi_{3}}{2\left(\tau_{1} \Xi_{1}+\tau_{2} \Xi_{2}\right)}\right) \\
& =\left(\frac{\prod_{s=1}^{2}\left(1+\mu^{\epsilon}\right)^{\omega_{s}}-\prod_{s=1}^{2}\left(1-\mu^{\epsilon}{ }_{s}\right)^{\omega_{s}}}{\prod_{s=1}^{2}\left(1+\mu^{\epsilon}{ }_{s}\right)^{\omega_{s}}+\prod_{s=1}^{2}\left(1-\mu^{\epsilon}\right)^{\omega_{s}}}, \frac{\prod_{s=1}^{2}\left(1+\mu^{\epsilon}{ }_{s}-\lambda_{s}\right)^{\omega_{s}}}{2\left(\prod_{s=1}^{2}\left(1+\mu^{\epsilon}\right)^{\omega_{s}}+\prod_{s=1}^{2}\left(1-\mu^{\epsilon}{ }_{s}\right)^{\omega_{s}}\right)},\right. \\
& \left.\frac{\prod_{s=1}^{2}\left(1+\mu^{\epsilon}{ }_{s}-v_{s}\right)^{\omega_{s}}}{2\left(\prod_{s=1}^{2}\left(1+\mu^{\epsilon}\right)^{\omega_{s}}+\prod_{s=1}^{2}\left(1-\mu^{\epsilon}\right)^{\omega_{s}}\right)}\right) .
\end{aligned}
$$

By this, theorem holds for $n=2$.

Now assume, theorem holds for $n=k$, i.e.,

$\operatorname{SVNEIWA}\left(\alpha_{1}, \alpha_{2}, \ldots, \alpha_{k}\right)$

$$
\begin{aligned}
= & \frac{\left(\prod_{s=1}^{k}\left(1+\mu^{\epsilon}{ }_{s}\right)^{\omega_{s}}-\prod_{s=1}^{k}\left(1-\mu^{\epsilon}{ }_{s}\right)^{\omega_{s}}\right.}{\prod_{s=1}^{k}\left(1+\mu^{\epsilon}{ }_{s}\right)^{\omega_{s}}+\prod_{s=1}^{k}\left(1-\mu^{\epsilon}{ }_{s}\right)^{\omega_{s}}}, \\
& \frac{\prod_{s=1}^{k}\left(1+\mu^{\epsilon}{ }_{s}-\lambda_{s}\right)^{\omega_{s}}}{2\left(\prod_{s=1}^{k}\left(1+\mu^{\epsilon}{ }_{s}\right)^{\omega_{s}}+\prod_{s=1}^{k}\left(1-\mu^{\epsilon}{ }_{s}\right)^{\omega_{s}}\right)}, \\
& \left.\frac{\prod_{s=1}^{k}\left(1+\mu^{\epsilon}{ }_{s}-v_{s}\right)^{\omega_{s}}}{2\left(\prod_{s=1}^{k}\left(1+\mu^{\epsilon}{ }_{s}\right)^{\omega_{s}}+\prod_{s=1}^{k}\left(1-\mu^{\epsilon}{ }_{s}\right)^{\omega_{s}}\right)}\right)
\end{aligned}
$$

Now, we have to prove it for $n=k+1$.

$\operatorname{SVNEIWA}\left(\alpha_{1}, \alpha_{2}, \ldots, \alpha_{k+1}\right)$

$=\operatorname{SVNEIWA}\left(\alpha_{1}, \alpha_{2}, \ldots, \alpha_{k}\right) \oplus \alpha_{k+1}^{\omega_{k+1}}$

$=\left(\frac{\prod_{s=1}^{k}\left(1+\mu^{\epsilon}\right)^{\omega_{s}}-\prod_{s=1}^{k}\left(1-\mu^{\epsilon}\right)^{\omega_{s}}}{\prod_{s=1}^{k}\left(1+\mu^{\epsilon}{ }_{s}\right)^{\omega_{s}}+\prod_{s=1}^{k}\left(1-\mu^{\epsilon}\right)^{\omega_{s}}}\right.$,

$$
\begin{aligned}
& \prod_{s=1}^{k}\left(1+\mu^{\epsilon}{ }_{s}-\lambda_{s}\right)^{\omega_{s}} \\
& \overline{2\left(\prod_{s=1}^{k}\left(1+\mu^{\epsilon}{ }_{s}\right)^{\omega_{s}}+\prod_{s=1}^{k}\left(1-\mu^{\epsilon}{ }_{s}\right)^{\omega_{s}}\right)}, \\
& \left.\frac{\prod_{s=1}^{k}\left(1+\mu_{s}^{\epsilon}-v_{s}\right)^{\omega_{s}}}{2\left(\prod_{s=1}^{k}\left(1+\mu^{\epsilon}\right)^{\omega_{s}}+\prod_{s=1}^{k}\left(1-\mu_{s}^{\epsilon}\right)^{\omega_{s}}\right)}\right) \\
& \oplus\left(\frac{\left(1+\mu_{k+1}^{\epsilon}\right)^{\omega_{k+1}}-\left(1-\mu_{k+1}^{\epsilon}\right)^{\omega_{k+1}}}{\left(1+\mu_{k+1}^{\epsilon}\right)^{\omega_{k+1}}+\left(1-\mu_{k+1}^{\epsilon}\right)^{\omega_{k+1}}},\right. \\
& \left(1+\mu_{k+1}^{\epsilon}-\lambda_{k+1}\right)^{\omega_{k+1}} \\
& \overline{2\left(\left(1+\mu^{\epsilon}{ }_{k+1}\right)^{\omega_{k+1}}+\left(1-\mu_{k+1}^{\epsilon}\right)^{\omega_{k+1}}\right)}, \\
& \left.\frac{\left(1+\mu_{k+1}^{\epsilon}-v_{k+1}\right)^{\omega_{k+1}}}{2\left(\left(1+\mu^{\epsilon}{ }_{k+1}\right)^{\omega_{k+1}}+\left(1-\mu_{k+1}^{\epsilon}\right)^{\left.\omega_{k+1}\right)}\right.}\right) \\
& =\left(\frac{\prod_{s=1}^{k+1}\left(1+\mu^{\epsilon}\right)^{\omega_{s}}-\prod_{s=1}^{k+1}\left(1-\mu^{\epsilon}{ }_{s}\right)^{\omega_{s}}}{\prod_{s=1}^{k+1}\left(1+\mu^{\epsilon}{ }_{s}\right)^{\omega_{s}}+\prod_{s=1}^{k+1}\left(1-\mu^{\epsilon}{ }_{s}\right)^{\omega_{s}}},\right. \\
& \frac{\prod_{s=1}^{k+1}\left(1+\mu^{\epsilon}{ }_{s}-\lambda_{s}\right)^{\omega_{s}}}{2\left(\prod_{s=1}^{k+1}\left(1+\mu^{\epsilon}{ }_{s}\right)^{\omega_{s}}+\prod_{s=1}^{k+1}\left(1-\mu^{\epsilon}{ }_{s}\right)^{\omega_{s}}\right)}, \\
& \left.\frac{\prod_{s=1}^{k+1}\left(1+\mu^{\epsilon}{ }_{s}-v_{s}\right)^{\omega_{s}}}{2\left(\prod_{s=1}^{k+1}\left(1+\mu^{\epsilon}{ }_{s}\right)^{\omega_{s}}+\prod_{s=1}^{k+1}\left(1-\mu^{\epsilon}{ }_{s}\right)^{\omega_{s}}\right)}\right) .
\end{aligned}
$$


Hence, result holds for $n=k+1$. In this way, we complete the proof using mathematical induction.

Corollary 4.4 Let $y_{s} \geq 0, w_{s} \geq 0$ and $\sum_{s=1}^{n} w_{s}=1$, then

$\prod_{s=1}^{n} y_{s}^{w_{s}} \leq \sum_{s=1}^{n} w_{s} y_{s}$

equality holds iff $y_{1}=y_{2}=\ldots=y_{n}$.

Theorem 4.5 Let $\alpha_{p}=\left\langle\mu_{p}^{\epsilon}, \lambda_{p}, v_{p}\right\rangle$ be the assemblage of SVNNs then SVNEIWA operator and SVNEWA operator fulfilled the following inequality

$\operatorname{SVNEIWA}\left(\alpha_{1}, \alpha_{2}, \ldots, \alpha_{n}\right) \leq \operatorname{SVNEWA}\left(\alpha_{1}, \alpha_{2}, \ldots, \alpha_{n}\right)$.

Proof Here, we omit the proof.

Example 4.6 Assume an assemblage of five SVNNs $\alpha_{1}=$ $\langle 0.65,0.00,0.15\rangle, \alpha_{2}=\langle 0.65,0.25,0.00\rangle, \alpha_{3}=\langle 0.45$, $0.25,0.10\rangle, \alpha_{4}=\langle 0.55,0.35,0.25\rangle$ and $\alpha_{5}=\langle 0.10,0.20$, $0.50\rangle$ with WV $\omega=(0.25,0.25,0.10,0.10,0.30)^{T}$. Then, we can aggregate these numbers as

$$
\begin{aligned}
& \frac{\prod_{s=1}^{5}\left(1+\mu_{s}^{\epsilon}\right)^{\omega_{s}}-\prod_{s=1}^{5}\left(1-\mu_{s}^{\epsilon}\right)^{\omega_{s}}}{\prod_{s=1}^{5}\left(1+\mu^{\epsilon}{ }_{s}\right)^{\omega_{s}}+\prod_{s=1}^{5}\left(1-\mu^{\epsilon}\right)^{\omega_{s}}}=0.483895, \\
& \frac{\prod_{s=1}^{5}\left(1+\mu_{s}^{\epsilon}-\lambda_{s}\right)^{\omega_{s}}}{2\left(\prod_{s=1}^{5}\left(1+\mu^{\epsilon}{ }_{s}\right)^{\omega_{s}}+\prod_{s=1}^{5}\left(1-\mu^{\epsilon}{ }_{s}\right)^{\omega_{s}}\right)}=0.320646, \\
& \frac{\prod_{s=1}^{5}\left(1+\mu^{\epsilon}{ }_{s}-v_{s}\right)^{\omega_{s}}}{2\left(\prod_{s=1}^{5}\left(1+\mu^{\epsilon}\right)^{\omega_{s}}+\prod_{s=1}^{5}\left(1-\mu^{\epsilon}\right)^{\omega_{s}}\right)}=0.294632
\end{aligned}
$$

and

$$
\begin{aligned}
\operatorname{SVNEIWA}\left(\alpha_{1}, \alpha_{2}, \ldots, \alpha_{5}\right) & \\
= & \left(\frac{\prod_{s=1}^{5}\left(1+\mu^{\epsilon}{ }_{s}\right)^{\omega_{s}}-\prod_{s=1}^{5}\left(1-\mu^{\epsilon}{ }_{s}\right)^{\omega_{s}}}{\prod_{s=1}^{5}\left(1+\mu^{\epsilon}{ }_{s}\right)^{\omega_{s}}+\prod_{s=1}^{5}\left(1-\mu^{\epsilon}{ }_{s}\right)^{\omega_{s}}},\right. \\
& \frac{\prod_{s=1}^{5}\left(1+\mu^{\epsilon}{ }_{s}-\lambda_{s}\right)^{\omega_{s}}}{2\left(\prod_{s=1}^{5}\left(1+\mu^{\epsilon}{ }_{s}\right)^{\omega_{s}}+\prod_{s=1}^{5}\left(1-\mu^{\epsilon}{ }_{s}\right)^{\omega_{s}}\right)}, \\
= & \langle 0.483895,0.320646,0.294632\rangle .
\end{aligned}
$$

\section{SVNEIOWA operator}

Definition 4.7 Let $\alpha_{p}=\left\langle\mu^{\epsilon}{ }_{p}, \lambda_{p}, v_{p}\right\rangle$ be the assemblage of SVNNs and (SVNEIOWA): $\Lambda^{n} \rightarrow \Lambda$ if,

$$
\begin{aligned}
& \text { SVNEIOWA }\left(\alpha_{1}, \alpha_{2}, \ldots, \alpha_{n}\right) \\
& =\bigoplus_{s=1}^{n} \omega_{s} \alpha_{£(s)}
\end{aligned}
$$

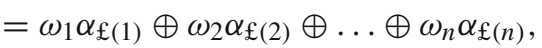

where $\omega=\left(\omega_{1}, \omega_{2}, \ldots, \omega_{n}\right)^{T}$ is the $\mathrm{WV}$ of $\left(\alpha_{1}, \alpha_{2}, \ldots, \alpha_{n}\right)$, such that $0 \leq \omega_{s} \leq 1$ and $\sum_{s=1}^{n} \omega_{s}=1 . £(1), £(2), \ldots, £(n)$

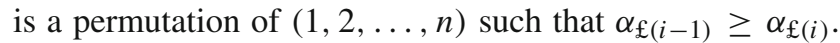
Then, the SVNEIOWG is called the single valued neutrosophic Einstein interactive ordered weighted averaging operator.

Theorem 4.8 Let $\alpha_{p}=\left\langle\mu_{p}^{\epsilon}, \lambda_{p}, v_{p}\right\rangle$ be the assemblage of SVNNs, then

$$
\begin{aligned}
& \operatorname{SVNEIOWA}\left(\alpha_{1}, \alpha_{2}, \ldots, \alpha_{n}\right)
\end{aligned}
$$

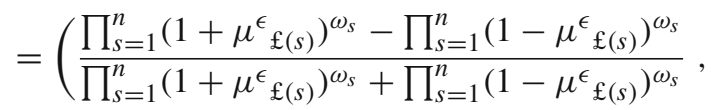

$$
\begin{aligned}
& \prod_{s=1}^{n}\left(1+\mu_{£(s)}^{\epsilon}-\lambda_{£(s)}\right)^{\omega_{s}} \\
& \overline{2\left(\prod_{s=1}^{n}\left(1+\mu_{£(s)}^{\epsilon}\right)^{\omega_{s}}+\prod_{s=1}^{n}\left(1-\mu_{£(s)}^{\epsilon}\right)^{\left.\omega_{s}\right)},\right.} \\
& \left.\frac{\prod_{s=1}^{n}\left(1+\mu_{£(s)}^{\epsilon}-v_{£(s)}\right)^{\omega_{s}}}{2\left(\prod_{s=1}^{n}\left(1+\mu_{£(s)}^{\epsilon}\right)^{\omega_{s}}+\prod_{s=1}^{n}\left(1-\mu_{£(s)}^{\epsilon}\right)^{\left.\omega_{s}\right)}\right.}\right) \text {. }
\end{aligned}
$$

Proof Proof is same as Theorem 4.3.

Example 4.9 Assume an assemblage of five SVNNs $\alpha_{1}=$ $\langle 0.65,0.00,0.15\rangle, \alpha_{2}=\langle 0.65,0.25,0.00\rangle, \alpha_{3}=\langle 0.45,0.25$, $0.10\rangle, \alpha_{4}=\langle 0.55,0.35,0.25\rangle$ and $\alpha_{5}=\langle 0.75,0.20,0.10\rangle$ with WV $\omega=(0.25,0.25,0.10,0.10,0.30)^{T}$. Then, we can aggregate these numbers as

$$
\begin{aligned}
& \frac{\prod_{s=1}^{5}\left(1+\mu_{£(s)}^{\epsilon}\right)^{\omega_{s}}-\prod_{s=1}^{5}\left(1-\mu_{£(s)}^{\epsilon}\right)^{\omega_{s}}}{\prod_{s=1}^{5}\left(1+\mu_{£(s)}^{\epsilon}\right)^{\omega_{s}}+\prod_{s=1}^{5}\left(1-\mu_{£(s)}^{\epsilon}\right)^{\omega_{s}}}=0.63430, \\
& \frac{\prod_{s=1}^{5}\left(1+\mu_{£(s)}^{\epsilon}-\lambda_{£(s)}\right)^{\omega_{s}}}{2\left(\prod_{s=1}^{5}\left(1+\mu_{£(s)}^{\epsilon}\right)^{\omega_{s}}+\prod_{s=1}^{5}\left(1-\mu_{£(s)}^{\epsilon}\right)^{\omega_{s}}\right)}=0.35432, \\
& \frac{\prod_{s=1}^{5}\left(1+\mu_{£(s)}^{\epsilon}-v_{£(s)}\right)^{\omega_{s}}}{2\left(\prod_{s=1}^{5}\left(1+\mu_{£(s)}^{\epsilon}\right)^{\omega_{s}}+\prod_{s=1}^{5}\left(1-\mu_{£(s)}^{\epsilon}\right)^{\omega_{s}}\right)}=0.37027
\end{aligned}
$$


and

$$
\begin{aligned}
& \operatorname{SVNEIOWA}\left(\alpha_{1}, \alpha_{2}, \ldots, \alpha_{5}\right)
\end{aligned}
$$

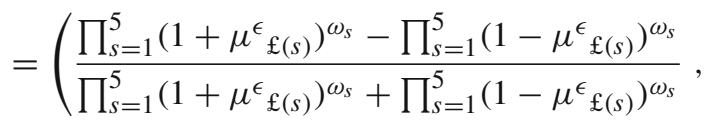

$$
\begin{aligned}
& \prod_{s=1}^{5}\left(1+\mu_{£(s)}^{\epsilon}-\lambda_{£(s)}\right)^{\omega_{s}}
\end{aligned}
$$

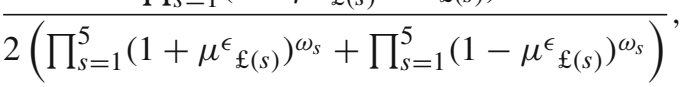

$$
\begin{aligned}
& \left.\frac{\prod_{s=1}^{5}\left(1+\mu_{£(s)}^{\epsilon}-v_{£(s)}\right)^{\omega_{s}}}{2\left(\prod_{s=1}^{5}\left(1+\mu_{£(s)}^{\epsilon}\right)^{\omega_{s}}+\prod_{s=1}^{5}\left(1-\mu^{\epsilon}{ }_{(s)}\right)^{\omega_{s}}\right)}\right) \\
& =\langle 0.63430,0.35432,0.37027\rangle \text {. }
\end{aligned}
$$

\section{SVNEIWG operator}

We will examine the weighted geometric AOs based on these improved Einstein procedures in the following manner.

Definition 4.10 Let $\alpha_{p}=\left\langle\mu_{p}^{\epsilon}, \lambda_{p}, v_{p}\right\rangle$ be the assemblage of SVNNs and (SVNEIWG): $\Lambda^{n} \rightarrow \Lambda$ if,

$$
\begin{aligned}
\operatorname{SVNEIWG}\left(\alpha_{1}, \alpha_{2}, \ldots, \alpha_{n}\right) & =\bigotimes_{s=1}^{n} \alpha_{s}^{\omega_{s}} \\
& =\alpha_{1}^{\omega_{1}} \otimes \alpha_{2}^{\omega_{2}} \otimes \ldots \otimes \alpha_{n}^{\omega_{n}},
\end{aligned}
$$

$\omega=\left(\omega_{1}, \omega_{2}, \ldots, \omega_{n}\right)^{T}$ is the $\mathrm{WV}$ of $\left(\alpha_{1}, \alpha_{2}, \ldots, \alpha_{n}\right)$, such that $0 \leq \omega_{s} \leq 1$ and $\sum_{s=1}^{n} \omega_{s}=1$ and where $\Lambda$ is the set of all SVNNs. Then, the SVNEIWG is called the singlevalued neutrosophic Einstein interactive weighted geometric (SVNEIWG) operator.

Theorem 4.11 Let $\alpha_{p}=\left\langle\mu_{p}^{\epsilon}, \lambda_{p}, v_{p}\right\rangle$ be the assemblage of SVNNs, then

$$
\begin{aligned}
& \text { SVNEIWG }\left(\alpha_{1}, \alpha_{2}, \ldots, \alpha_{n}\right) \\
& =\left(\frac{\prod_{s=1}^{n}\left(1+v_{s}-\mu_{s}^{\epsilon}\right)^{\omega_{s}}}{2\left(\prod_{s=1}^{n}\left(1+v_{s}\right)^{\omega_{s}}+\prod_{s=1}^{n}\left(1-v_{s}\right)^{\omega_{s}}\right)},\right. \\
& \frac{\prod_{s=1}^{n}\left(1+\lambda_{s}\right)^{\omega_{s}}-\prod_{s=1}^{n}\left(1-\lambda_{s}\right)^{\omega_{s}}}{\prod_{s=1}^{n}\left(1+\lambda_{s}\right)^{\omega_{s}}+\prod_{s=1}^{n}\left(1-\lambda_{s}\right)^{\omega_{s}}}, \\
& \left.\frac{\prod_{s=1}^{n}\left(1+v_{s}\right)^{\omega_{s}}-\prod_{s=1}^{n}\left(1-v_{s}\right)^{\omega_{s}}}{\prod_{s=1}^{n}\left(1+v_{s}\right)^{\omega_{s}}+\prod_{s=1}^{n}\left(1-v_{s}\right)^{\omega_{s}}}\right) .
\end{aligned}
$$

Proof Proof is same as Theorem 4.3.

Theorem 4.12 Let $\alpha_{p}=\left\langle\mu_{p}^{\epsilon}, v_{p}\right\rangle$ be the assemblage of SVNNs then SVNEWIG operator and SVNEWG operator fulfilled the following inequality

$\operatorname{SVNEIWG}\left(\alpha_{1}, \alpha_{2}, \ldots, \alpha_{n}\right) \geq \operatorname{SVNEWG}\left(\alpha_{1}, \alpha_{2}, \ldots, \alpha_{n}\right)$.
Example 4.13 Assume an assemblage of five SVNNs $\alpha_{1}=$ $\langle 0.65,0.00,0.15\rangle, \alpha_{2}=\langle 0.65,0.25,0.00\rangle, \alpha_{3}=\langle 0.45,0.25$, $0.10\rangle, \alpha_{4}=\langle 0.55,0.35,0.25\rangle$ and $\alpha_{5}=\langle 0.10,0.20,0.50\rangle$ with WV $\omega=(0.25,0.25,0.10,0.10,0.30)^{T}$. Then, we can aggregate these numbers as

$$
\begin{aligned}
& \frac{\prod_{s=1}^{5}\left(1+v_{s}-\mu_{s}^{\epsilon}\right)^{\omega_{s}}}{2\left(\prod_{s=1}^{5}\left(1+v_{s}\right)^{\omega_{s}}+\prod_{s=1}^{5}\left(1-v_{s}\right)^{\omega_{s}}\right.}=0.168941, \\
& \frac{\prod_{s=1}^{5}\left(1+\lambda_{s}\right)^{\omega_{s}}-\prod_{s=1}^{5}\left(1-\lambda_{s}\right)^{\omega_{s}}}{\prod_{s=1}^{5}\left(1+\lambda_{s}\right)^{\omega_{s}}+\prod_{s=1}^{5}\left(1-\lambda_{s}\right)^{\omega_{s}}}=0.184617, \\
& \frac{\prod_{s=1}^{5}\left(1+v_{s}\right)^{\omega_{s}}-\prod_{s=1}^{5}\left(1-v_{s}\right)^{\omega_{s}}}{\prod_{s=1}^{5}\left(1+v_{s}\right)^{\omega_{s}}+\prod_{s=1}^{5}\left(1-v_{s}\right)^{\omega_{s}}}=0.233749
\end{aligned}
$$

and

$$
\begin{aligned}
& \operatorname{SVNEIWG}\left(\alpha_{1}, \alpha_{2}, \ldots, \alpha_{5}\right) \\
&=\left(\frac{\prod_{s=1}^{5}\left(1+v_{s}-\mu_{s}^{\epsilon}\right)^{\omega_{s}}}{2\left(\prod_{s=1}^{5}\left(1+v_{s}\right)^{\omega_{s}}+\prod_{s=1}^{5}\left(1-v_{s}\right)^{\omega_{s}}\right)},\right. \\
& \frac{\prod_{s=1}^{5}\left(1+\lambda_{s}\right)^{\omega_{s}}-\prod_{s=1}^{5}\left(1-\lambda_{s}\right)^{\omega_{s}}}{\prod_{s=1}^{5}\left(1+\lambda_{s}\right)^{\omega_{s}}+\prod_{s=1}^{5}\left(1-\lambda_{s}\right)^{\omega_{s}}}, \\
&\left.\frac{\prod_{s=1}^{5}\left(1+v_{s}\right)^{\omega_{s}}-\prod_{s=1}^{5}\left(1-v_{s}\right)^{\omega_{s}}}{\prod_{s=1}^{5}\left(1+v_{s}\right)^{\omega_{s}}+\prod_{s=1}^{5}\left(1-v_{s}\right)^{\omega_{s}}}\right) \\
&=\langle 0.168941,0.184617,0.233749\rangle .
\end{aligned}
$$

\section{SVNEIOWG operator}

Definition 4.14 Let $\alpha_{p}=\left\langle\mu_{p}^{\epsilon}, \lambda_{p}, v_{p}\right\rangle$ be the assemblage of SVNNs and (SVNEIOWG): $\Lambda^{n} \rightarrow \Lambda$ if,

$$
\begin{aligned}
& \text { SVNEIOWG }\left(\alpha_{1}, \alpha_{2}, \ldots, \alpha_{n}\right)=\bigotimes_{s=1}^{n} \alpha_{\mathfrak{f}(s)}^{\omega_{s}} \\
& =\alpha_{\mathfrak{f}(1)}^{\omega_{1}} \otimes \alpha_{\mathfrak{f}(2)}^{\omega_{2}} \otimes \ldots \otimes \alpha_{\mathfrak{f}(n)}^{\omega_{n}},
\end{aligned}
$$

where $\omega=\left(\omega_{1}, \omega_{2}, \ldots, \omega_{n}\right)^{T}$ is the $\mathrm{WV}$ of $\left(\alpha_{1}, \alpha_{2}, \ldots, \alpha_{n}\right)$, such that $0 \leq \omega_{s} \leq 1$ and $\sum_{s=1}^{n} \omega_{s}=1 . £(1), £(2), \ldots, £(n)$ is a permutation of $(1,2, \ldots, n)$ such that $\alpha_{\mathfrak{f}(i-1)} \geq \alpha_{\mathfrak{f}(i)}$. Then, the SVNEIOWG is called the single-valued neutrosophic Einstein interactive ordered weighted geometric operator. 
Theorem 4.15 Let $\alpha_{p}=\left\langle\mu_{p}^{\epsilon}, \lambda_{p}, v_{p}\right\rangle$ be the assemblage of SVNNs, then

$$
\begin{aligned}
& \text { SVNEIOWG }\left(\alpha_{1}, \alpha_{2}, \ldots, \alpha_{n}\right) \\
& =\left(\frac{\prod_{s=1}^{n}\left(1+v_{s}-\mu_{s}^{\epsilon}\right)^{\omega_{\mathfrak{f}(s)}}}{2\left(\prod_{s=1}^{n}\left(1+v_{s}\right)^{\omega_{\mathfrak{f}(s)}}+\prod_{s=1}^{n}\left(1-v_{s}\right)^{\left.\omega_{\mathfrak{f}(s)}\right)}\right.},\right. \\
& \quad \frac{\prod_{s=1}^{n}\left(1+\lambda_{s}\right)^{\omega_{\mathfrak{f}(s)}}-\prod_{s=1}^{n}\left(1-\lambda_{s}\right)^{\omega_{\mathfrak{f}(s)}}}{\prod_{s=1}^{n}\left(1+\lambda_{s}\right)^{\omega_{\mathfrak{f}(s)}}+\prod_{s=1}^{n}\left(1-\lambda_{s}\right)^{\omega_{\mathfrak{f}(s)}}}, \\
& \\
& \left.\quad \frac{\prod_{s=1}^{n}\left(1+v_{s}\right)^{\omega_{\mathfrak{E}(s)}}-\prod_{s=1}^{n}\left(1-v_{s}\right)^{\omega_{\mathfrak{E}(s)}}}{\prod_{s=1}^{n}\left(1+v_{s}\right)^{\omega_{\mathfrak{f}(s)}}+\prod_{s=1}^{n}\left(1-v_{s}\right)^{\omega_{\mathfrak{f}(s)}}}\right) .
\end{aligned}
$$

Proof Proof is same as Theorem 4.3.

Example 4.16 Assume an assemblage of five SVNNs $\alpha_{1}=$ $\langle 0.65,0.00,0.15\rangle, \alpha_{2}=\langle 0.65,0.25,0.00\rangle, \alpha_{3}=\langle 0.45,0.25$, $0.10\rangle, \alpha_{4}=\langle 0.55,0.35,0.25\rangle$ and $\alpha_{5}=\langle 0.75,0.20,0.10\rangle$ with $\mathrm{WV} \omega=(0.25,0.25,0.10,0.10,0.30)^{T}$. Then, we can aggregate these numbers as

$$
\begin{aligned}
& \frac{\prod_{s=1}^{5}\left(1+v_{s}-\mu_{s}^{\epsilon}\right)^{\omega_{\mathfrak{E}(s)}}}{2\left(\prod_{s=1}^{5}\left(1+v_{s}\right)^{\omega_{\mathfrak{f}(s)}}+\prod_{s=1}^{5}\left(1-v_{s}\right)^{\omega_{\mathfrak{E}(s)}}\right)}=0.125688, \\
& \frac{\prod_{s=1}^{5}\left(1+\lambda_{s}\right)^{\omega_{\mathfrak{E}(s)}}-\prod_{s=1}^{5}\left(1-\lambda_{s}\right)^{\omega_{\mathfrak{E}(s)}}}{\prod_{s=1}^{5}\left(1+\lambda_{s}\right)^{\omega_{\mathfrak{E}(s)}}+\prod_{s=1}^{5}\left(1-\lambda_{s}\right)^{\omega_{\mathfrak{E}(s)}}}=0.208305, \\
& \frac{\prod_{s=1}^{5}\left(1+v_{s}\right)^{\omega_{\mathfrak{f}(s)}}-\prod_{s=1}^{5}\left(1-v_{s}\right)^{\omega_{\mathfrak{f}(s)}}}{\prod_{s=1}^{5}\left(1+v_{s}\right)^{\omega_{\mathfrak{E}(s)}}+\prod_{s=1}^{5}\left(1-v_{s}\right)^{\omega_{\mathfrak{E}(s)}}}=0.148422
\end{aligned}
$$

and

$$
\begin{aligned}
& \operatorname{SVNEIWG}\left(\alpha_{1}, \alpha_{2}, \ldots, \alpha_{5}\right) \\
&=\left(\frac{\prod_{s=1}^{5}\left(1+v_{s}-\mu_{s}^{\epsilon}\right)^{\omega_{\mathfrak{f}(s)}}}{2\left(\prod_{s=1}^{5}\left(1+v_{s}\right)^{\omega_{\mathfrak{f}(s)}}+\prod_{s=1}^{5}\left(1-v_{s}\right)^{\omega_{\mathfrak{f}(s)}}\right)},\right. \\
& \\
& \quad \frac{\prod_{s=1}^{5}\left(1+\lambda_{s}\right)^{\omega_{\mathfrak{f}(s)}}-\prod_{s=1}^{5}\left(1-\lambda_{s}\right)^{\omega_{\mathfrak{f}(s)}}}{\prod_{s=1}^{5}\left(1+\lambda_{s}\right)^{\omega_{\mathfrak{f}(s)}}+\prod_{s=1}^{5}\left(1-\lambda_{s}\right)^{\omega_{\mathfrak{f}(s)}}} \\
&\left.\frac{\prod_{s=1}^{5}\left(1+v_{s}\right)^{\omega_{\mathfrak{f}(s)}}-\prod_{s=1}^{5}\left(1-v_{s}\right)^{\omega_{\mathfrak{f}(s)}}}{\prod_{s=1}^{5}\left(1+v_{s}\right)^{\omega_{\mathfrak{f}(s)}}+\prod_{s=1}^{5}\left(1-v_{s}\right)^{\omega_{\mathfrak{f}(s)}}}\right) \\
&=\langle 0.125688,0.208305,0.148422\rangle .
\end{aligned}
$$

\section{Proposed approach for MCDM}

Assume an assemblage of alternatives denoted by $\left\{\zeta_{\rho_{1}}^{\tau}, \zeta_{\rho_{2}}^{\tau}\right.$, $\left.\ldots, \zeta_{\rho_{n}}^{\tau}\right\}$ and $C=\left\{\eta_{\varrho_{1}}^{\delta}, \eta_{\varrho_{2}}^{\delta}, \ldots, \eta_{\varrho_{m}}^{\delta}\right\}$ is the assemblage of criteria. DM evaluates all alternatives under the different criteria. SVNNs are used by DMs to express their preferences, $\alpha_{i j}=\left\langle\mu_{i j}^{\epsilon}, v_{i j}\right\rangle$ s.t $0 \leq \mu^{\epsilon}{ }_{i j}, v_{i j} \leq 1$ and $\mu^{\epsilon q}{ }_{i j}+v_{i j}^{q} \leq 1$ for $i=1,2, \ldots, n ; j=1,2, \ldots, m$. If all Performance criteria are the same kind, there is no need for normalization; however, since MCDM has two different types of Evaluation criteria (benefit kind attributes $\tau_{b}$ and cost kinds attributes $\tau_{c}$ ), the matrix $D$ has been transformed into a normalize matrix using the normalization formula $Y=\left(\beth_{i j}^{\Upsilon}\right)_{m \times n}$,

$\left(\beth_{i j}^{\Upsilon}\right)_{m \times n}= \begin{cases}\left(\mathscr{B}_{i j}\right)^{c} ; & j \in \tau_{c} \\ \mathscr{B}_{i j} ; & j \in \tau_{b},\end{cases}$

where $\left(\mathscr{B}_{i j}\right)^{c}$ show the compliment of $\mathscr{B}_{i j}$.

The suggested operators will be implemented to the MCDM, which will require the preceding steps.

\section{Algorithm}

Input: Decision matrix and Weight vector.

Output: Optimal alternative.

1:

Obtain the decision matrix $D=\left(\mathscr{B}_{i j}\right)_{m \times n}$ in the format of SVNNs from DM.

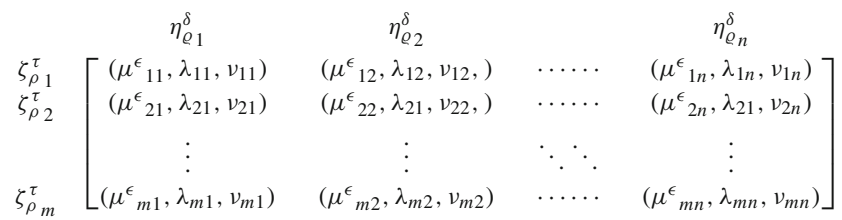

2:

There is no need for normalization if all indicators are of the same kind, but in MCGDM, there may be two types of criteria. The matrix was updated to the transforming response matrix in this case $Y=\left(\beth_{i j}^{\Upsilon}\right)_{m \times n}$ using the normalization formula Eq. 25.

3:

Using the DM information and the suggested operator given in Eqs. 26 and 27, aggregate the overall values $\beth_{i j}^{\Upsilon}(j=$ $1,2, \ldots, m)$ of the alternative $\zeta_{\rho_{i}}^{\tau}$ to the collective one $\beth_{i}^{\Upsilon}(i=$ $1,2, \ldots, n)$.

$$
\begin{aligned}
& \operatorname{SVNEIWA}\left(\alpha_{1}, \alpha_{2}, \ldots, \alpha_{n}\right) \\
& =\frac{\left(\prod_{s=1}^{n}\left(1+\mu_{s}^{\epsilon}\right)^{\omega_{s}}-\prod_{s=1}^{n}\left(1-\mu^{\epsilon}\right)^{\omega_{s}}\right.}{\prod_{s=1}^{n}\left(1+\mu^{\epsilon}\right)^{\omega_{s}}+\prod_{s=1}^{n}\left(1-\mu^{\epsilon}\right)^{\omega_{s}}}, \\
& \frac{\prod_{s=1}^{n}\left(1+\mu^{\epsilon}{ }_{s}-\lambda_{s}\right)^{\omega_{s}}}{2\left(\prod_{s=1}^{n}\left(1+\mu^{\epsilon}\right)^{\omega_{s}}+\prod_{s=1}^{n}\left(1-\mu^{\epsilon}\right)^{\omega_{s}}\right)}, \\
& \left.\frac{\prod_{s=1}^{n}\left(1+\mu^{\epsilon}-v_{s}\right)^{\omega_{s}}}{2\left(\prod_{s=1}^{n}\left(1+\mu_{s}^{\epsilon}\right)^{\omega_{s}}+\prod_{s=1}^{n}\left(1-\mu^{\epsilon}\right)^{\omega_{s}}\right)}\right) .
\end{aligned}
$$

$\operatorname{SVNEIWG}\left(\alpha_{1}, \alpha_{2}, \ldots, \alpha_{n}\right)$

$$
=\left(\frac{\prod_{s=1}^{n}\left(1+v_{s}-\mu^{\epsilon}\right)^{\omega_{s}}}{2\left(\prod_{s=1}^{n}\left(1+v_{s}\right)^{\omega_{s}}+\prod_{s=1}^{n}\left(1-v_{s}\right)^{\omega_{s}}\right)},\right.
$$


Fig. 1 Procedural steps of Algorithm

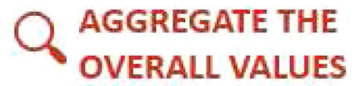

OVERALL VALUES

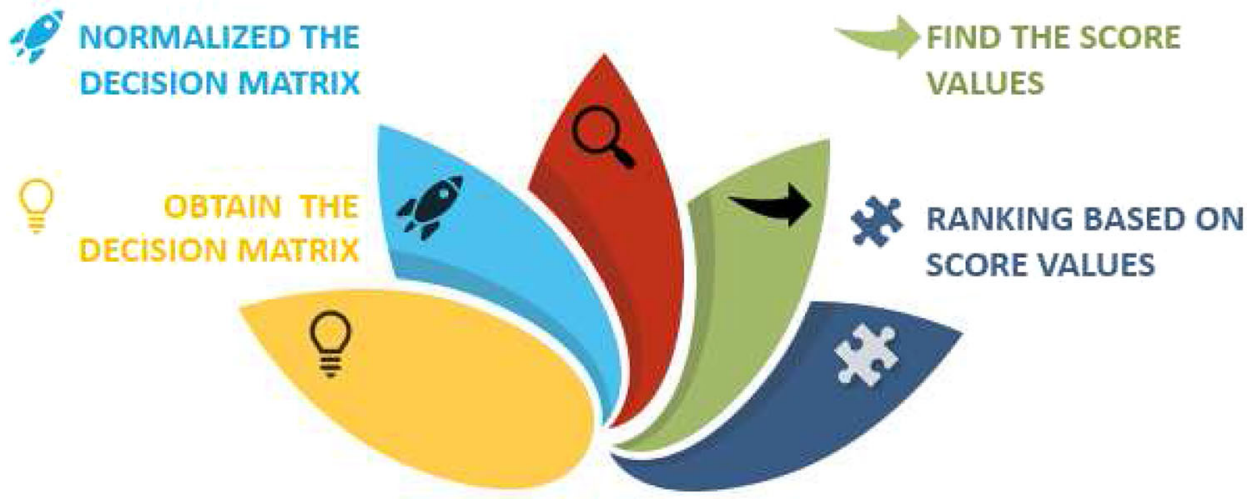

$$
\begin{aligned}
& \frac{\prod_{s=1}^{n}\left(1+\lambda_{s}\right)^{\omega_{s}}-\prod_{s=1}^{n}\left(1-\lambda_{s}\right)^{\omega_{s}}}{\prod_{s=1}^{n}\left(1+\lambda_{s}\right)^{\omega_{s}}+\prod_{s=1}^{n}\left(1-\lambda_{s}\right)^{\omega_{s}}}, \\
& \left.\frac{\prod_{s=1}^{n}\left(1+v_{s}\right)^{\omega_{s}}-\prod_{s=1}^{n}\left(1-v_{s}\right)^{\omega_{s}}}{\prod_{s=1}^{n}\left(1+v_{s}\right)^{\omega_{s}}+\prod_{s=1}^{n}\left(1-v_{s}\right)^{\omega_{s}}}\right) .
\end{aligned}
$$

\section{4:}

The score value of each aggregated value is computed by using definition of score function.

5:

Order the alternatives according to their score value and choose the best one (s).

Pictorial view of Algorithm is given in Fig. 1.

\section{Case study}

According to the Intergovernmental Panel on Climate Change (IPCC), the exceptionally rapid environmental degradation is very certainly the product of human activities [70]. The resultant climate change has substantial environmental consequences, including the extinction of animal species [71], decreasing agricultural productivity [72], more extreme weather patterns [73], and human immigration [74]. There is growing momentum to cut global greenhouse gas (GHG) emissions in order to slow the path of changing climate. For example recently, France passed a bill requiring a $40 \%$ decrease in GHG emissions by 2030 compared to 1990 [75]. However the use of carbon fuels is also not the only producer of GHG it is by far the most significant. According to the U.S environmental, protection agency fossil fuels account for $76 \%$ of all human-caused emissions in the U.S [76]. It is reasonable to infer that a considerable reduction in GHG emissions implies a reduction in the use of fossil fuels. However, this is not an easy undertaking because products generated from hydrocarbons are not just energy carriers, but also primary energy sources. Hydrogen is an energy source that, in order to have a truly significant impact on decarbonization, should be created in an environmentally friendly manner. In 2017, fossil fuels accounted for more than 85 percent of worldwide energy production [77]. As a result, if the world totally transitioned to a hydrogen economy that eliminated all fossil fuel consumption, an energy deficit would develop immediately. This element provides a substantial challenge in terms of locating suitable power sources [78]. This topic, however, will not be addressed in this study.

Since humanity is approaching the "end of the cheap oil era," there is widespread agreement in the science and energy sectors that a new energy carrier must be discovered. Various countries' rigorous down-selection processes revealed that hydrogen will be the eventual choice. Hydrogen might be used as a supplementary energy source in the automobile industry without emitting any $\mathrm{CO}_{2}$ by utilizing technological breakthroughs such as hydrogen fuel cells to provide power for an automated transmission as well as a direct fuel for internal combustion engines. One of its factors for the favor for hydrogen is the variety of its generation feedstock. Because there is almost no plentiful hydrogen gas in nature, the only option is to free it from its chemical bonds with other atoms. There are essentially two methods for producing hydrogen, among others: split hydrocarbons or splitting water. Steam fermentation is used to break down hydrocarbons. Water splitting can be accomplished directly at extreme temperatures or with the use of electricity. Another method for creating hydrogen from water is to burn coal in the presence of water vapor [79].

It makes sense to transform fossilized waste fuels like natural gas first into hydrogen and then use it in fuel cell vehicles. Finally, when fossil fuels become prohibitively expensive and, most likely, illegally contribute to global warming, renewable primary energies will enter the picture, either for financial or ecological reasons. In terms of energy content, weight, and volume, the newly produced hydrogen 


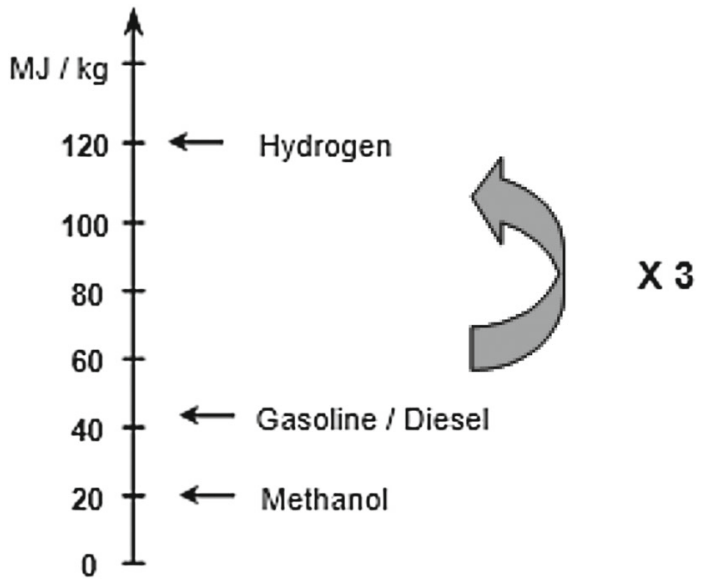

Fig. 2 Gravimetric energy density of different fuels

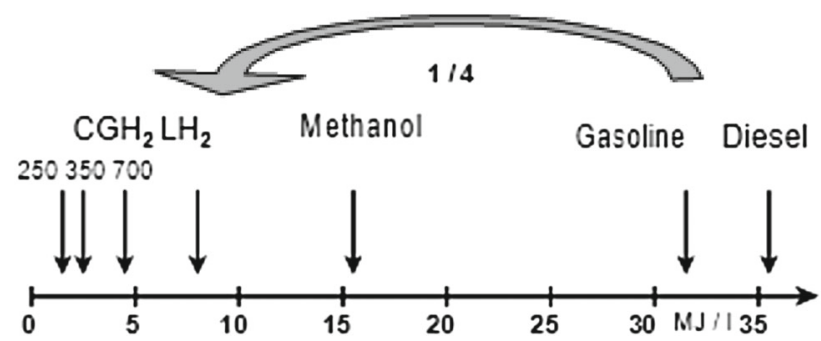

Fig. 3 Volumetric energy density of different fuels

fuel differs significantly from the commonly used ones. The lightweight of hydrogen in comparison to its energy capacity is the most notable feature, as depicted in Fig. 2. The energy content of hydrogen gas per $\mathrm{kg}$ is $120 \mathrm{MJ}$, which is three times that of gasoline and diesel fuel. The advantage over methanol is sixfold [80].

In contrast to its outstanding gravimetric density, hydrogen has a low volumetric energy density. The volumetric density of hydrogen is determined by its aggregation state. Even pressures of up to 700 bar are insufficient to deal with the great characteristics of hydrocarbons such as gasoline and diesel. Only liquid hydrogen can attain a fair price that is still less than a quarter of the price of gasoline, as shown in Fig. 3. As a result, hydrogen tanks for automotive applications will take up far more area than liquid hydrocarbon tanks currently in use [81].

Cryogenic storage tanks are also known as cryogenic storage containers. The Dewar (named after James Dewar, the inventor of liquid oxygen and hydrogen storage) is practically a double-walled super-insulators container, as seen in Fig. 4. It transports liquid oxygen, nitrogen, hydrogen, helium, and argon gas at a temperature of $<110 \mathrm{~K} / 163{ }^{\circ} \mathrm{C}$. Liquid hydrogen is already recognized as a superior energy source. Because water is solely an exhaust product when transformed into electricity, it is non-toxic and incredibly environmentally friendly.
The materials used in cryogenic container design are generally dictated by safety and economy [82]. The fundamental difficulty in the case of a cryogenic container is the safety concerns, and the design specifications under the context of low-temperature embrittlement can be described as follows:

- Fracture toughness: The boiling point of liquefied nitrogen gas is around $-196{ }^{\circ} \mathrm{C}$, while that of liquefied hydrogen gas is approximately $-253{ }^{\circ} \mathrm{C}$. At this level, the materials losing their ductility and becomes brittle. As a result, materials must be robust enough to withstand brittle fracture. Metals with a face-centered cube (FCC) lattice are appropriate due to their insensitivity at low temperatures. All nickel-copper alloys, aluminum and its alloys, and austenitic stainless steels containing more than $7 \%$ nickel are all acceptable for building cryogenic storage containers [83].

- Heat transfer: The passage of heat through the cryogenic tank's wall is mainly conduction. Materials with low thermal conductance are preferred.

- Thermal stress: Because of the low temperature, the inner wall contracts, causing thermal strains. As a result, materials with a low thermal conductivity are appropriate.

- Thermal diffusivity: In practise, comprehensive thermal insulating is not conceivable. Materials should be chosen in such a way that they can dissipate heat as soon as possible. Diffusivity is a measurement of the rate of heat transmission, which is inversely related to the specific heat of the material.

- Transportation: Transportation is possible with materials with a lower specific gravity.

Material selection in any field of engineering is very important stage of designing. Engineering design is driven by the goals of performance, cost, and environmental sensitivity, and is generally constrained by materials. The purpose of optimum product design is to select materials that best fit the criteria of the design while providing maximum performance at the lowest possible cost. Material selection is decision-making process by considering many conflicting criterion. AOs play a vital role in decision-making. In some circumstances, it has been discovered that the current Einstein AOs appear hesitant to make the correct option during the decision-making process. These AOs must be updated to address these specific issues. We propose some novel operations and the AOs that go with them for aggregating various SVNNs. Our presented model outperforms other models.

According to the above-mentioned explanation and MC DM's perspective, all features can be classified, given in Table 2.

The case study was conducted in an automotive parts manufacturing company in Malaysia, and the study was conducted for an automotive component, cryogenic storage 


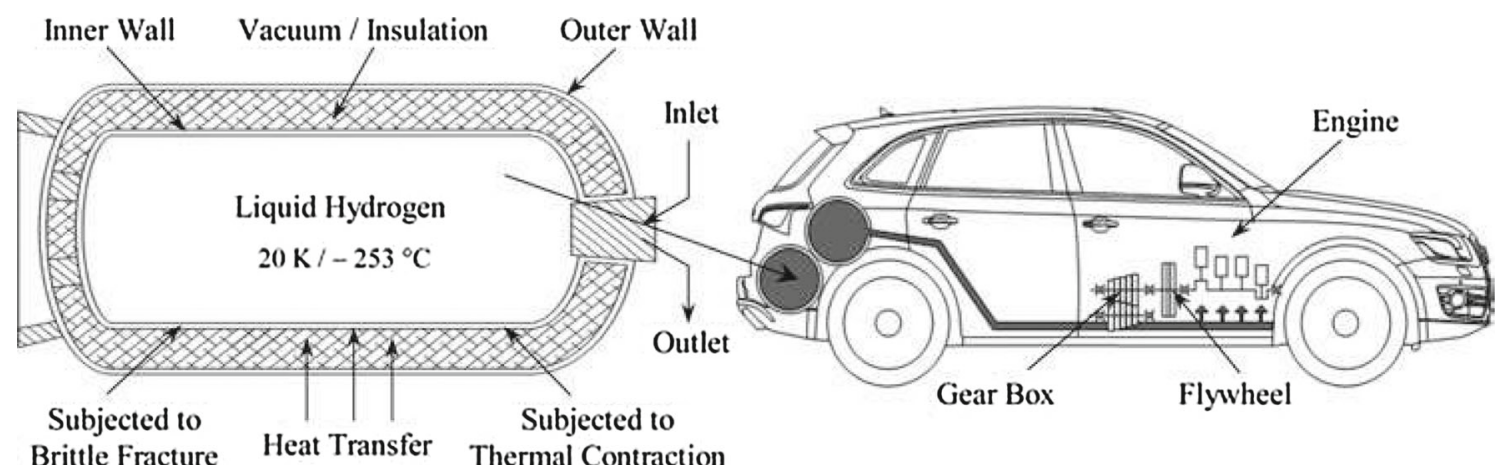

Fig. 4 Cryogenic storage container

Table 2 Material selection criterion for cryogenic storage

\begin{tabular}{ll}
\hline$\eta_{\varrho_{1}}^{\delta}$ & Specific gravity \\
$\eta_{\varrho_{2}}^{\delta}$ & Toughness index \\
$\eta_{\varrho_{3}}^{\delta}$ & Yield stress \\
$\eta_{\varrho_{4}}^{\delta}$ & Young's modulus \\
$\eta_{\varrho_{5}}^{\delta}$ & Easily accessible \\
\hline
\end{tabular}

container. As part of the process of applying sustainability concepts, the company must choose a suitable material for the parts produced. It focuses on the cryogenic storage container first, then on the other parts. The inputs for the weights of the parameters and material were gathered from the DMs. SVN theory and proposed AOs have been employed to overcome the complexity and indecisiveness of human judgement. The materials are chosen with the three fundamental pillars of sustainability in mind: the material should be economical, environmentally friendly, and useful to society. The most important factors (parameters) to consider while choosing material for an instrumental panel by DMs, five feasible parameters given in Table 2. The selection process begins with a preliminary screening of materials that can be utilized for instrument panels, taking into account the features inherent to the application. During screening, the material kinds that are potentially applicable are identified. It is critical to determine the material types that can be used for the instrumental panel early in the material selection process. Four materials are screened after analyzing the qualities: $\zeta_{\rho_{1}}^{\tau}=\mathrm{Ti}-6 \mathrm{Al}-4 \mathrm{~V}, \zeta_{\rho_{2}}^{\tau}=\mathrm{SS} 301-\mathrm{FH}, \zeta_{\rho_{3}}^{\tau}=70 \mathrm{Cu}-30 \mathrm{Zn}$, and $\zeta_{\rho_{4}}^{\tau}=$ Inconel 718 . Let the $\mathrm{WV}$ of these parameters is $\omega=(0.25,0.25,0.10,0.10,0.30)^{T}$ obtained from the normal distribution method.

\section{By SVNEIWA operator}

\section{Step 1:}

Obtain the decision matrix $D=\left(\mathscr{B}_{i j}\right)_{m \times n}$ in the format of SVNNs from DM. The judgement values are described in Table 3.

\section{Step 2:}

Normalize the decision matrices acquired by DM using Equ. 25 in this case only $\eta_{\varrho_{1}}^{\delta}$ is cost type criteria, others are benefits type criteria. Normalized matrix is given in Table 4.

\section{Step 3:}

Using SVNEIWA operator, aggregate the overall values $\beth_{i j}^{\Upsilon}(j=1,2, \ldots, m)$ of the alternative $\zeta_{\rho_{i}}^{\tau}$ to the collective one $\beth_{i}^{\Upsilon}(i=1,2, \ldots, n)$.

$$
\begin{aligned}
& \beth_{1}^{\Upsilon}=\operatorname{SVNEIWA}\left(\beth_{11}^{\Upsilon}, \beth_{12}^{\Upsilon}, \beth_{13}^{\Upsilon}, \beth_{14}^{\Upsilon}, \beth_{15}^{\Upsilon}\right) \\
& =(0.406188,0.317279,0.263274) ; \\
& \beth_{2}^{\Upsilon}=\operatorname{SVNEIWA}\left(\beth_{21}^{\Upsilon}, \beth_{22}^{\Upsilon}, \beth_{23}^{\Upsilon}, \beth_{24}^{\Upsilon}, \beth_{25}^{\Upsilon}\right) \\
& =(0.572886,0.338951,0.316215) ; \\
& \beth_{3}^{\Upsilon}=\operatorname{SVNEIWA}\left(\beth_{31}^{\Upsilon}, \beth_{32}^{\Upsilon}, \beth_{33}^{\Upsilon}, \beth_{34}^{\Upsilon}, \beth_{35}^{\Upsilon}\right) \\
& =(0.264534,0.260505,0.239028) ; \\
& \beth_{4}^{\Upsilon}=\operatorname{SVNEIWA}\left(\beth_{41}^{\Upsilon}, \beth_{42}^{\Upsilon}, \beth_{43}^{\Upsilon}, \beth_{44}^{\Upsilon}, \beth_{45}^{\Upsilon}\right) \\
& =(0.341426,0.251602,0.244883) .
\end{aligned}
$$

\section{Step 4:}

The suggested SVNEIWA operator has been implemented based on this information, and therefore the consolidated SVNNs relating to the given alternate, the ranking arrangement of the alternatives dependent on the score values is $\zeta_{\rho_{2}}^{\tau} \succ \zeta_{\rho_{4}}^{\tau} \succ \zeta_{\rho_{3}}^{\tau} \succ \zeta_{\rho_{1}}^{\tau}$, where the optimal alternative is same as the initial decision-making ranking. As a result, the proposed methodology meets the first test condition.

Compute the score for all SVNN aggregated values $\beth_{i}^{\Upsilon}$.

$$
\begin{aligned}
& \breve{\Xi}\left(\beth_{1}^{\Upsilon}\right)=0.608545 ; \\
& \breve{\Xi}\left(\beth_{2}^{\Upsilon}\right)=0.639240 ; \\
& \breve{\Xi}\left(\beth_{3}^{\Upsilon}\right)=0.588334 ; \\
& \breve{\Xi}\left(\beth_{4}^{\Upsilon}\right)=0.614980 .
\end{aligned}
$$

\section{Step 5:}

Ranks according to score values.

$\beth_{2}^{\Upsilon} \succ \beth_{4}^{\Upsilon} \succ \beth_{1}^{\Upsilon} \succ \beth_{3}^{\Upsilon}$. 
Table 3 Assessment matrix acquired from DM

\begin{tabular}{llllll}
\hline & $\eta_{\varrho_{1}}^{\delta}$ & $\eta_{\varrho_{2}}^{\delta}$ & $\eta_{\varrho_{3}}^{\delta}$ & $\eta_{\varrho_{4}}^{\delta}$ & $\eta_{\ell_{5}}^{\delta}$ \\
\hline$\zeta_{\rho_{1}}^{\tau}$ & $(0.25,0.15,0.10)$ & $(0.45,0.10,0.50)$ & $(0.55,0.15,0.40)$ & $(0.65,0.25,0.10)$ & $(0.45,0.10,0.35)$ \\
$\zeta_{\rho_{2}}^{\tau}$ & $(0.35,0.25,0.60)$ & $(0.55,0.25,0.20)$ & $(0.55,0.15,0.40)$ & $(0.75,0.15,0.20)$ & $(0.50,0.20,0.35)$ \\
$\zeta_{\rho_{3}}^{\tau}$ & $(0.25,0.25,0.55)$ & $(0.15,0.25,0.50)$ & $(0.45,0.15,0.35)$ & $(0.15,0.15,0.20)$ & $(0.05,0.20,0.15)$ \\
$\zeta_{\rho_{4}}^{\tau}$ & $(0.20,0.20,0.25)$ & $(0.20,0.35,0.40)$ & $(0.65,0.500 .25)$ & $(0.35,0.40,0.25)$ & $(0.45,0.35,0.50)$ \\
\hline
\end{tabular}

Table 4 Normalized matrix acquired from DM

\begin{tabular}{llllll}
\hline & $\eta_{\varrho_{1}}^{\delta}$ & $\eta_{\varrho_{2}}^{\delta}$ & $\eta_{\varrho_{3}}^{\delta}$ & $\eta_{\varrho_{4}}^{\delta}$ & $\eta_{\varrho_{5}}^{\delta}$ \\
\hline$\zeta_{\rho_{1}}^{\tau}$ & $(0.10,0.15,0.25)$ & $(0.45,0.10,0.50)$ & $(0.55,0.15,0.40)$ & $(0.65,0.25,0.10)$ & $(0.45,0.10,0.35)$ \\
$\zeta_{\rho_{2}}^{\tau}$ & $(0.60,0.25,0.35)$ & $(0.55,0.25,0.20)$ & $(0.55,0.15,0.40)$ & $(0.75,0.15,0.20)$ & $(0.50,0.20,0.35)$ \\
$\zeta_{\rho_{3}}^{\tau}$ & $(0.55,0.25,0.25)$ & $(0.15,0.25,0.50)$ & $(0.45,0.15,0.35)$ & $(0.15,0.15,0.20)$ & $(0.05,0.20,0.15)$ \\
$\zeta_{\rho_{4}}^{\tau}$ & $(0.25,0.20,0.20)$ & $(0.20,0.35,0.40)$ & $(0.65,0.500 .25)$ & $(0.35,0.40,0.25)$ & $(0.45,0.35,0.50)$ \\
\hline
\end{tabular}

Table 5 Modified decision matrix

\begin{tabular}{llllll}
\hline & $\eta_{\varrho_{1}}^{\delta}$ & $\eta_{\varrho_{2}}^{\delta}$ & $\eta_{\varrho_{3}}^{\delta}$ & $\eta_{\varrho_{4}}^{\delta}$ & $\eta_{\varrho_{5}}^{\delta}$ \\
\hline$\zeta_{\rho_{1}}^{\tau}$ & $(0.10,0.15,0.25)$ & $(0.45,0.10,0.50)$ & $(0.55,0.15,0.40)$ & $(0.65,0.25,0.10)$ & $(0.45,0.10,0.35)$ \\
$\zeta_{\rho_{2}}^{\tau}$ & $(0.60,0.25,0.35)$ & $(0.55,0.25,0.20)$ & $(0.55,0.15,0.40)$ & $(0.75,0.15,0.20)$ & $(0.50,0.20,0.35)$ \\
$\zeta_{\rho_{3}}^{\tau}$ & $(0.25,0.25,0.55)$ & $(0.50,0.25,0.15)$ & $(0.35,0.15,0.45)$ & $(0.20,0.15,0.15)$ & $(0.15,0.20,0.05)$ \\
$\zeta_{\rho_{4}}^{\tau}$ & $(0.20,0.20,0.25)$ & $(0.40,0.35,0.20)$ & $(0.25,0.500 .65)$ & $(0.25,0.40,0.35)$ & $(0.50,0.35,0.45)$ \\
\hline
\end{tabular}

So,

$\zeta_{\rho_{2}}^{\tau} \succ \zeta_{\rho_{4}}^{\tau} \succ \zeta_{\rho_{1}}^{\tau} \succ \zeta_{\rho_{3}}^{\tau}$

Because material evaluation is began at the theoretical phase of the project, there is more scope to examine the appropriateness of the selected material at the implementation stage.
FCC materials are commonly employed at low temperatures of $-163^{\circ} \mathrm{C}$. The austenitic steel SS301-FH is ranked first, which is consistent with past research and real-world practise. Austenitic steel is still commonly used in liquid nitrogen or hydrogen storage containers [84].

In some cases, the second option is extremely important in the ranking. The titanium alloy (Ti-6Al-4V) came in sec-
Table 6 Comparison of proposed operators with some exiting operators

\begin{tabular}{|c|c|c|c|}
\hline Authors & $\mathrm{AOs}$ & Ranking of alternatives & The optimal alternative \\
\hline \multirow[t]{2}{*}{ Wu et al. [46] } & SNNPWA & $\zeta_{\rho_{2}}^{\tau} \succ \zeta_{\rho_{1}}^{\tau} \succ \zeta_{\rho_{4}}^{\tau} \succ \zeta_{\rho_{3}}^{\tau}$ & $\zeta_{\rho_{2}}^{\tau}$ \\
\hline & SNNPWG & $\zeta_{\rho_{2}}^{\tau} \succ \zeta_{\rho_{4}}^{\tau} \succ \zeta_{\rho_{3}}^{\tau} \succ \zeta_{\rho_{1}}^{\tau}$ & $\zeta_{\rho_{2}}^{\tau}$ \\
\hline \multirow[t]{2}{*}{ Peng et al. [37] } & SNNWA & $\zeta_{\rho_{2}}^{\tau} \succ \zeta_{\rho_{3}}^{\tau} \succ \zeta_{\rho_{1}}^{\tau} \succ \zeta_{\rho_{4}}^{\tau}$ & $\zeta_{\rho 2}^{\tau}$ \\
\hline & SNNWG & $\zeta_{\rho_{2}}^{\tau} \succ \zeta_{\rho_{4}}^{\tau} \succ \zeta_{\rho_{1}}^{\tau} \succ \zeta_{\rho_{3}}^{\tau}$ & $\zeta_{\rho_{2}}^{\tau}$ \\
\hline \multirow[t]{2}{*}{ Garg and Nancy [50] } & SVNPMM & $\zeta_{\rho_{2}}^{\tau} \succ \zeta_{\rho_{4}}^{\tau} \succ \zeta_{\rho_{1}}^{\tau} \succ \zeta_{\rho_{3}}^{\tau}$ & $\zeta_{\rho_{2}}^{\tau}$ \\
\hline & SVNPDMM & $\zeta_{\rho_{2}}^{\tau} \succ \zeta_{\rho_{3}}^{\tau} \succ \zeta_{\rho_{4}}^{\tau} \succ \zeta_{\rho_{1}}^{\tau}$ & $\zeta_{\rho 2}^{\tau}$ \\
\hline \multirow[t]{2}{*}{ Wei and Wei [48] } & SVNDPWA & $\zeta_{\rho_{2}}^{\tau} \succ \zeta_{\rho_{1}}^{\tau} \succ \zeta_{\rho_{4}}^{\tau} \succ \zeta_{\rho_{3}}^{\tau}$ & $\zeta_{\rho_{2}}^{\tau}$ \\
\hline & SVNDPWG & $\zeta_{\rho_{2}}^{\tau} \succ \zeta_{\rho_{4}}^{\tau} \succ \zeta_{\rho_{1}}^{\tau} \succ \zeta_{\rho_{3}}^{\tau}$ & $\zeta_{\rho_{2}}^{\tau}$ \\
\hline \multirow[t]{2}{*}{ Liu [49] } & SVNNWA & $\zeta_{\rho_{2}}^{\tau} \succ \zeta_{\rho_{3}}^{\tau} \succ \zeta_{\rho_{1}}^{\tau} \succ \zeta_{\rho_{4}}^{\tau}$ & $\zeta_{\rho_{2}}^{\tau}$ \\
\hline & SVNNWG & $\zeta_{\rho_{2}}^{\tau} \succ \zeta_{\rho_{4}}^{\tau} \succ \zeta_{\rho_{1}}^{\tau} \succ \zeta_{\rho_{3}}^{\tau}$ & $\zeta_{\rho_{2}}^{\tau}$ \\
\hline Li et al. [47] & GSNNEWA & $\zeta_{\rho_{2}}^{\tau} \succ \zeta_{\rho_{4}}^{\tau} \succ \zeta_{\rho_{3}}^{\tau} \succ \zeta_{\rho_{1}}^{\tau}$ & $\zeta_{\rho_{2}}^{\tau}$ \\
\hline \multirow[t]{2}{*}{ Proposed } & SVNEIWA & $\zeta_{\rho_{2}}^{\tau} \succ \zeta_{\rho_{4}}^{\tau} \succ \zeta_{\rho_{1}}^{\tau} \succ \zeta_{\rho_{3}}^{\tau}$ & $\zeta_{\rho_{2}}^{\tau}$ \\
\hline & SVNEIWG & $\zeta_{\rho_{2}}^{\tau} \succ \zeta_{\rho_{4}}^{\tau} \succ \zeta_{\rho_{3}}^{\tau} \succ \zeta_{\rho_{1}}^{\tau}$ & $\zeta_{\rho_{2}}^{\tau}$ \\
\hline
\end{tabular}


ond place in the articles of Dehghan-Manshadi et al. [85] and Jahan et al. [86]. Although Ti-6Al-4V is strong in the aerospace sector, titanium alloys are relatively poor in lowtemperature embrittlement scenarios, whereas Inconel is a better cryogenic storage tank material than titanium alloy.

\section{Authenticity analysis}

To illustrate the validity of the proposed technique, Wang and Triantaphyllou [?] validated the following test criteria:

1. Test 1: If we substitute the rating values of the nonoptimal alternate with those of the worse option, the optimum alternate should not vary as long as the respective WV remain constant.

2. Test 2: The structure of the approach should be transitive.

3. Test 3: When a discrete problem is subdivided and the same MCDM technique is applied, the aggregated rating of the alternates should be the same as the evaluation of the original problem.

In the section below, we confirmed the conditions given on our suggested MCDM methodology.

\section{Authenticity test 1}

Under this test, if we exchange the MDs and NMDs of alternatives $\zeta_{\rho 1}^{\tau}$ and $\zeta_{\rho 3}^{\tau}$ in the Table 4 , the modified decision matrix as shown below in Table 5 .

\section{Authenticity test 2 and test 3}

If we breakdown the provided problem into the sub-problems $\left\{\zeta_{\rho_{1}}^{\tau}, \zeta_{\rho_{2}}^{\tau}\right\},\left\{\zeta_{\rho_{2}}^{\tau}, \zeta_{\rho_{3}}^{\tau}\right\},\left\{\zeta_{\rho_{3}}^{\tau}, \zeta_{\rho_{4}}^{\tau}\right\}$ and $\left\{\zeta_{\rho_{4}}^{\tau}, \zeta_{\rho_{1}}^{\tau}\right\}$ then apply the procedure steps of the proposed technique, we receive the ranking order of these smaller problems as $\zeta_{\rho_{2}}^{\tau} \succeq \zeta_{\rho_{1}}^{\tau}$, $\zeta_{\rho_{2}}^{\tau} \succeq \zeta_{\rho_{3}}^{\tau}, \zeta_{\rho_{4}}^{\tau} \succeq \zeta_{\rho_{3}}^{\tau}$ and $\zeta_{\rho_{4}}^{\tau} \succeq \zeta_{\rho_{1}}^{\tau}$. As a result of merging them, the total ranking order of the alternate is $\zeta_{\rho_{2}}^{\tau} \succ \zeta_{\rho_{4}}^{\tau} \succ$ $\zeta_{\rho_{1}}^{\tau} \succ \zeta_{\rho 3}^{\tau}$, which is the same as the original ranking order. As a result, the proposed methodology meets authenticity test requirements 2 and 3.

\section{Comparison analysis}

In this section, we compare recommended operators to specific present AOs. The fact that both achieve the same outcome illustrates the superiority of our proposed AOs. By resolving the information data with certain existing AOs, we compare our results and arrive at the same ideal conclusion. This indicates the resilience and consistency of the paradigm we proposed. In the existing AOs, there is no impact of TMD on IMD and FMD. We found that by modifying the degrees of the TMDs of the SVNNs in the Einstein AOs for SVNNs, the collective IMDs and FMDs of the SVNEWA operator remain independently of its. this is the main disadvantages of all existing AOs. We obtain $\zeta_{\rho_{2}}^{\tau} \succ \zeta_{\rho_{4}}^{\tau} \succ \zeta_{\rho_{3}}^{\tau} \succ \zeta_{\rho_{1}}^{\tau}$ rating by our proposed aggregation operators; to validate our optimal option, we run this problem through other existing AOs. The validity of our suggested aggregation operators is demonstrated by the fact that we obtain the same optimal decision. Table 6 provides a comparison of the AOs offered with some existing AOs.

\section{Conclusion}

In engineering design, delicate balance of shape, appropriate material, and construction involve a wide range of problems. Mathematical modeling in engineering design balance the use of all resources while satisfying design objectives under economic, quality, and safety limitations under uncertainty. To make the best judgement, the problem should be precisely organized in accordance with the decision requirements. In real decision-making, the evaluation data for alternatives offered by decision makers (DMs) is typically vague, imprecise, and inconsistent; hence, SVNSs can be used to process such uncertain information. Einstein operators are well-known AOs for smooth approximation. It has been observed that the existing Einstein AOs are apprehensive to make the optimal decision during the decision-making process in some circumstances. In order to deal with complexities in material selection, an innovative aggregation technique is proposed for material selection in engineering design based on truthness, indeterminacy, and falsity indexes of SVNSs. Taking advantages of SVNSs and smooth approximation with interactive Einstein operations, singlevalued neutrosophic Einstein interactive weighted averaging and geometric operators are proposed named as "singlevalued neutrosophic Einstein interactive weighted averaging (SVNEIWA) operator", "single-valued neutrosophic Einstein interactive ordered weighted averaging (SVNEIOWA) operator", "single-valued neutrosophic Einstein interactive weighted geometric (SVNEIWG) operator" and "singlevalued neutrosophic Einstein interactive ordered weighted geometric (SVNEIOWG) operator". Additionally, we proposed new MCDM methodology to demonstrate the efficiency and applicability of the suggested AOs.

The proposed model works efficiently when the input is SVNNs. However, with some minor changes, the proposed model can be extended to handle other types of the input data. Based on proposed AOs, a robust MCDM approach is proposed for material selection in engineering design. A practical application of proposed MCDM approach for material selection of cryogenic storage container is developed. Additionally, the authenticity analysis and comparison anal- 
ysis are designed to discuss validity and rationality of the optimal decision.

The suggested work has a wide range of potential applications. This model can be used in further research for bid evaluation for construction-contractor selection, evaluating passenger happiness, information fusion, information management, information aggregation, information measures, statistical approaches, machine learning, neural networks, medical diagnosis, artificial intelligence, and computational intelligence.

Open Access This article is licensed under a Creative Commons Attribution 4.0 International License, which permits use, sharing, adaptation, distribution and reproduction in any medium or format, as long as you give appropriate credit to the original author(s) and the source, provide a link to the Creative Commons licence, and indicate if changes were made. The images or other third party material in this article are included in the article's Creative Commons licence, unless indicated otherwise in a credit line to the material. If material is not included in the article's Creative Commons licence and your intended use is not permitted by statutory regulation or exceeds the permitted use, you will need to obtain permission directly from the copyright holder. To view a copy of this licence, visit http://creativecomm ons.org/licenses/by/4.0/.

\section{References}

1. Chatterjee P, Chakraborty S (2012) Material selection using preferential ranking methods. Mater Design 35(3):84-93

2. Thakker A, Jarvis J, Buggy M, Sahed A (2008) A novel approach to materials selection strategy case study: wave energy extraction impulse turbine blade. Mater Design 29(19):73-80

3. Edwards KL (2011) Materials influence on design: a decade of development. Mater Design 32(10):73-80

4. Prashant RG, Gupta N (2010) Material selection for microelectronic heat sinks: an application of the Ashby approach. Mater Design 31(11):3-7

5. Farag MM (2008) Quantitative methods of materials substitution: application to automotive components. Mater Design 29(3):74-80

6. Rao RV (2008) A decision making methodology for material selection using an improved compromise ranking method. Mater Design 29(19):49-54

7. Roth R, Field F, Clark J (1994) Materials selection and multiattribute utility analysis. J. Comput.-Aided Mater. Design 1(3):2542

8. Kutz M, (2002) Handbook of material selection, Jhon Willey \& Sons

9. Ashby MF, Johnson K (2002) Materials and design: the art and science of material selection in product design. ButterworthHeinemann, Burlington

10. Kesteren IEHV, Stappers PJ, de Bruij JCM. http://www.nordes.org/ data/uploads/papers/88.pdf

11. Crilly N, Moultrie J, Clarkson PJ (2004) Seeing things: consumer response to the visual domain in product design. Design Stud 25(6):547-577

12. Karana E, Hekkert P, Kandachar P (2009) Meanings of materials through sensorial properties and manufacturing processes. Mater Design 30(7):2778-2784

13. Changxu S (1998) Materials and sustainable development. J Shanghai Univ $2: 2-7$
14. Bahraminasab M, Jahan A (2011) Material selection for femoral component of total knee replacement using comprehensive VIKOR. Mater Design 32:4471-4477

15. Holloway L (1998) Materials selection for optimal environmental impact in mechanical design. Mater Design 19(4):133-143

16. Jacobs P, Sadler B (1989) Sustainable development and environmental assessment: perspectives on planning for a common future. Canadian Environmental Assessment Research Council, Quebec

17. Chatterjee P, Athawale VM, Chakraborty S (2011) Materials selection using complex proportional assessment and evaluation of mixed data methods. Mater Design 32:851-860

18. Chatterjee P, Athawale VM, Chakraborty S (2009) Selection of materials using compromise ranking and outranking methods. Mater Design 30:4043-4053

19. Yeh CH (2002) A problem-based selection of multi-attribute decision-making methods. Int Trans Operat Res 9:169-181

20. Voogd H (1983) Multicriteria evaluation for urban and regional planning. Pion Ltd., London

21. Hajkowicz S, Collins K (2007) A review of multiple criteria analysis for water resource planning and management. Water Resour Manage 21:1553-1566

22. Shanian A, Savadogo O (2006) A material selection model based on the concept of multiple attribute decision making. Mater Design 27:329-37

23. Shanian A, Savadogo O (2006) TOPSIS multiple-criteria decision support analysis for material selection of metallic bipolar plates for polymer electrolyte fuel cell. J Power Sour 159:1095-1104

24. Rao RV (2006) A material selection model using graph theory and matrix approach. Mater Sci Eng A 431:248-255

25. Chan JWK (2006) Application of grey relational analysis for ranking material options. Int J Comput Appl Technol 26:210-217

26. Chan JWK, Tong TKL (2007) Multi-criteria material selections and end-of-life product strategy: grey relational analysis approach. Mater Design 28:1539-1546

27. Bovea MD, Gallardo A (2006) The influence of impact assessment methods on materials selection for eco-design. Mater Design 27:209-215

28. Giachetti RE (1998) A decision support system for material and manufacturing process selection. J Intell Manuf 9:265-276

29. Thurston DL, Carnahan JV (1992) Fuzzy ratings and utility analysis in preliminary design evaluation of multiple attributes. J Mech Design 114:648-658

30. Wang MJJ, Chang TC (1995) Tool steel materials selection under fuzzy environment. Fuzzy Sets Syst 72:263-270

31. Liao TW (1996) A fuzzy multicriteria decision-making method for material selection. J Manuf Syst 15:1-12

32. Chen SM (1997) A new method for tool steel materials selection under fuzzy environment. Fuzzy Sets Syst 92:265-74

33. Zadeh LA (1965) Fuzzy sets. Inform Control 8:338-353

34. Atanassov KT (1986) Intuitionistic fuzzy sets. Fuzzy Sets Syst 20(1):87-96

35. Smarandache F (1998) Neutrosophy neutrosophic probability, set, and logic, ProQuest information and learning. LearnQuest, Ann Arbor

36. Wang H, Smarandache F, Zhang YQ, Sunderraman R (2010) Single valued neutrosophic sets. Multispace Multistruct 4:410-413

37. Peng JJ, Wang JQ, Wang J, Zhang HY, Chen ZH (2016) Simplified neutrosophic sets and their applications in multi-criteria group decision-making problems. Int J Syst Sci 47:2342-2358

38. Wang H, Smarandache F, Zhang YQ, Smarandache R (2005) Interval Neutrosophic Sets and Logic: Theory and Applications In Computing. Hexis, Phoenix

39. Nancy, Garg H (2016) An improved score function for ranking neutrosophic sets and its application to decision-making process. Int J Uncertainty Quantif 6(5):377-385 
40. Garg H, Nancy (2017) Some new biparametric distance measures on single-valued neutrosophic sets with applications to pattern recognition and medical diagnosis. Information 8:162

41. Ye J (2014) A multicriteria decision-making method using aggregation operators for simplified neutrosophic sets. J Intell Fuzzy Syst 26:2459-2466

42. Peng JJ, Wang JQ, Wang J, Zhang HY, Chen ZH (2016) Simplified neutrosophic sets and their applications in multi-criteria group decision-making problems. Int J Syst Sci 47:2342-2358

43. Nancy, Garg H (2016) Novel single-valued neutrosophic decision making operators under Frank norm operations and its application. Int J Uncertain Quantif 6:361-375

44. Liu P, Chu Y, Li Y, Chen Y (2014) Some generalized neutrosophic number hamacher aggregation operators and their application to group decision making. Int J Fuzzy Syst 16:242-255

45. Zhang HY, Wang JQ, Chen XH (2014) Interval neutrosophic sets and their application in multicriteria decision making problems. Sci World J 2014:645953

46. Wu XH, Wang JQ, Peng JJ, Chen XH (2016) Cross-entropy and prioritized aggregation operator with simplified neutrosophic sets and their application in multi-criteria decision-making problems, International Journal of Fuzzy. System 18:1104-1116

47. Li B, Wang J, Yang L, Li A X (2018) Novel generalized simplified neutrosophic number Einstein aggregation operator. Int J Appl Math 48(1):1-6

48. Wei G, Wei Y (2018) Some single-valued neutrosophic dombi prioritized weighted aggregation operators in multiple attribute decision making. J Intell Fuzzy Syst 35(2):2001-2013

49. Liu P (2016) The aggregation operators based on archimedean tConorm and t-Norm for single-valued neutrosophic numbers and their application to decision making. Int J Fuzzy Syst 18(5):849863

50. Garg H, Nancy (2018) Multi-criteria decision-making method based on prioritized muirhead mean aggregation operator under neutrosophic set environment. Symmetry 10:280

51. Wang J, Tang X, Wei G (2018) Models for multiple attribute decision-making with dual generalized single-valued neutrosophic bonferroni mean operators. Algorithms 11(1):1-15

52. Mondal K, Pramanik S, Giri BC, Smarandache F (2018) NNHarmonic mean aggregation operators-based MCGDM strategy in a neutrosophic number environment. Axioms 7(1):1-16

53. Ji P, Wang JQ, Zhang HY (2018) Frank prioritized Bonferroni mean operator with single-valued neutrosophic sets and its application in selecting third-party logistics providers. Neural Comput Appl 30(3):799-823

54. Wei G, Zhang Z (2019) Some single-valued neutrosophic bonferroni power aggregation operators in multiple attribute decision making. J Ambient Intell Humaniz Comput 10(3):863-882

55. Yang Y, Chen ZS, Rodriguez RM, Pedrycz W, Chin KS (2021) Novel fusion strategies for continuous interval-valued q-rung orthopair fuzzy information: a case study in quality assessment of SmartWatch appearance design. Int J Mach Learn Cybern. https:// doi.org/10.1007/s13042-020-01269-2

56. Chen ZS, Yu C, Chin kS, Martinez L (2019) An enhanced ordered weighted averaging operators generation algorithm with applications for multicriteria decision making. Appl Math Model 71:467-490

57. Chen ZS, Yang LL, Rodriguez RM, Xiong SH, Chin KS, Martinez L (2021) Power-average-operator-based hybrid multiattribute online product recommendation model for consumer decisionmaking. Int J Intell Syst. https://doi.org/10.1002/int.22394

58. Chen ZS, Liu XL, Chin KS, Pedrycz W, Tsui KL, Skibniewski MJ (2021) Online-review analysis based large-scale group decisionmaking for determining passenger demands and evaluating passenger satisfaction: Case study of high-speed rail system in China. Inf Fusion 69:22-39
59. Chen ZS, Zhang X, Rodríguez RM, Pedrycz W, Martínez L (2021) Expertise-based bid evaluation for construction-contractor selection with generalized comparative linguistic ELECTRE III. Autom Constr 125:103578

60. Riaz M, Hashmi MR (2019) Linear Diophantine fuzzy set and its applications towards multi-attribute decision making problems. J Intell Fuzzy Syst 37(4):5417-5439

61. Riaz M, Farid HMA, Aslam M, Pamucar D, Bozanic D (2021) Novel approach for third-party reverse logistic provider selection process under linear Diophantine fuzzy prioritized aggregation operators. Symmetry 13(7):1152

62. Iampan A, Garcia GS, Riaz M, Farid HMA, Chinram R (2021) Linear diophantine fuzzy Einstein aggregation operators for multicriteria decision-making problems. J Math 2021:5548033

63. Liu P, Liu J (2018) Some q-rung orthopai fuzzy bonferroni mean operators and their application to multi-attribute group decision making. Int J Int Syst 33(2):315-347

64. Riaz M, Garg H, Farid HMA, Chinram R (2021) Multi-criteria decision making based on bipolar picture fuzzy operators and new distance measures. Comput Model Eng Sci. https://doi.org/ $10.32604 / \mathrm{cmes} .2021 .014174$

65. Liu Z, Wang S, Liu P (2018) Multiple attribute group decision making based on q-rung orthopair fuzzy Heronianmean operators. Int J Intell Syst 33(12):2341-2363

66. Riaz M, Garg H, Farid HMA, Aslam M (2021) Novel q-rung orthopair fuzzy interaction aggregation operators and their application to low-carbon green supply chain management. J Intell Fuzzy Syst. https://doi.org/10.3233/JIFS-210506

67. Riaz M, Hamid MT, Farid HMA, Afzal D (2020) TOPSIS, VIKOR and aggregation operators based on q-rung orthopair fuzzy soft sets and their applications. J Intell Fuzzy Syst 39(5):6903-6917

68. Ye J, Zhan J, Xu ZS (2021) A novel multi-attribute decision-making method based on fuzzy rough sets. Comput Ind Eng 155:107136

69. Mu Z, Zeng S, Wang P (2021) Novel approach to multi-attribute group decision-making based on interval-valued Pythagorean fuzzy power Maclaurin symmetric mean operator. Comput Ind Eng 155:107049

70. Stocker TF, Qin D, Plattner GK, Tignor M, Allen SK, Boschung J, Nauels A, Xia Y, Bex V, Midgley PM (2013) ummary for Policymakers. In Climate Change, (2013) The physical science basis. Contribution of working group I to the fifth assessment report of the intergovernmental panel on climate change. Cambridge University Press, Cambridge

71. CaraDonna PJ, Cunningham JL, Iler AM (2018) Experimental warming in the field delays phenology and reduces body mass, fat content and survival: Implications for the persistence of a pollinator under climate change. Funct Ecol 32:2345-2356

72. Kontgis C, Schneider A, Ozdogan M, Kucharik C, Tri VPD, Duc NH, Schatz J (2019) Climate change impacts on rice productivity in the Mekong River Delta. Appl Geogr 102:71-83

73. Mazdiyasni O, AghaKouchak A (2015) Substantial increase in concurrent droughts and heatwaves in the United States. Procd Natl Acad Sci USA 112:11484-11489

74. Warner K, Ehrhart C, Sherbinin AD, Adamo S, Chai-Onn T (2009) In search of shelter: mapping the efects of climate change on human migration and displacement. Climate Change CARE International, London

75. AFHYPAC and FNCCR. Déployer les stations hydrogène dans votre territoire. (2018). Available online: http://www.afhypac.org/ documents/divers/GUIDE-STATION-HYDROGENE-WEB.pdf

76. United States Environmental Protection Agency. Inventory of U.S. Greenhouse Gas Emissions and Sinks: 1990-2015. (2017). Available online: https://www.epa.gov/sites/production/files/2017-02/ documents/2017complete-report.pdf

77. BP Statistical Review of World Energy. (2018). Available online: https://www.bp.com/content/dam/bp/en/corporate/pdf/energy- 
economics/statistical-review/bp-stats-review-2018-full-report. pdf

78. Saito $\mathrm{S}$ (2010) Role of nuclear energy to a future society of shortage of energy resources and global warming. J Nuclear Mater 398:1-9

79. Well-to-Wheel Energy Use and Greenhouse Gas Emissions of Advanced Fuel/Vehicle Systems - North American Analysis. General Motors, Argonne Nat. Lab., BP, Exxon Mobil, and Shell, (2001). Part 1: http://www.transportation.anl.gov/pdfs/TA/163. pdf Part 2: http://www.transportation.anl.gov/pdfs/TA/164.pdf

80. GM Well-to-Wheels Analysis of Energy Use and Greenhouse Gas Emissions of Advanced Fuels/Vehicles Systems. A European study. LBST, September (2002). http://www.lbst.de/gm-wtw

81. WELL-TO-WHEELS ANALYSIS OF FUTURE AUTOMOTIVE FUELS AND POWERTRAINS IN THE EUROPEAN CONTEXT, Version 1b. concawe, EUCAR, JRC, (January 2004). http://ies.jrc. cec.eu.int/Download/eh/31

82. Farag MM (2014) Materials and process selection for engineering design. CRC Press, Boca Raton

83. Flynn TM (2005) Cryogenic engineering. Marcel Dekker, New York

84. Godula-Jopek A, JehleWandWellnitz J (2012) Hydrogen storage technologies: new materials, transport, and infrastructure. Wiley$\mathrm{VCH}$, Boschstr
85. Dehghan-Manshadi B, MahmudiHand A (2007) A novel method for materials selection in mechanical design: combination of nonlinear normalization and a modified digital logic method. Mater Design 28:8-15

86. Jahan A, Bahraminasab M, Edwards KL (2012) A target-based normalization technique for material selection. Mater Design $35: 647-654$

Publisher's Note Springer Nature remains neutral with regard to jurisdictional claims in published maps and institutional affiliations. 